\title{
Enantioselective Synthesis of Linear Polypropionate Arrays Using Anthracene-Tagged Organosilanes
}

\author{
Sarathy Kesavan, Qibin Su, Jian Shao, John A. Porco, Jr. and James S. Panek \\ Department of Chemistry and Center for Chemical Methodology and Library \\ Development, 590 Commonwealth Avenue, Boston University, Boston, \\ Massachusetts 02215
}

\section{Supporting Information}

General Information: ${ }^{1} \mathrm{H}$ NMR spectra were recorded on a $400 \mathrm{MHz}$ spectrometer at ambient temperature with $\mathrm{CDCl}_{3}$ as the solvent unless otherwise stated. ${ }^{13} \mathrm{C}$ NMR spectra were recorded at $100 \mathrm{MHz}$ with complete proton decoupling at ambient temperature. Chemical shifts are reported in parts per million relative to chloroform $\left({ }^{1} \mathrm{H}, \delta 7.24 ;{ }^{13} \mathrm{C}, \delta 77.00\right)$. Data for ${ }^{1} \mathrm{H} \mathrm{NMR}$ are reported as follows: chemical shift, multiplicity, integration $(\operatorname{app}=$ apparent, par obsc $=$ partially obscure, ovrlp $=$ overlapping, $\mathrm{s}=$ singlet, $\mathrm{d}=$ doublet, $\mathrm{t}=$ triplet, $\mathrm{q}=$ quartet, $\mathrm{m}=$ multiplet $)$ and coupling constants. Infrared spectra were recorded on a Nicolet Nexus 670 FT-IR spectrophotometer. Optical rotations were recorded on an AUTOPOL III digital polarimeter at $589 \mathrm{~nm}$ and are recorded as $[\alpha] \mathrm{D}$ (concentration in grams $/ 100 \mathrm{~mL}$ solvent). HPLC-ELSD-MS analysis was performed on a Waters HPLC-MS system equipped with Waters 600 HPLC pump, Waters 2996 photodiode array detector, Micromass ZQ Quadrupole Mass Spectrometer, and Sedere Sedex 75 ELS detector (XTerra ${ }^{\circledR} \mathrm{RP} 8,5 \mu \mathrm{m}, 4.6 \times 30 \mathrm{~mm}$; Atlantis ${ }^{\mathrm{TM}} \mathrm{dC} 18,5 \mu \mathrm{m}, 4.6 \times 50 \mathrm{~mm}$ ). Low and high-resolution mass spectra were obtained in the Boston University Mass Spectrometry Laboratory using a Finnegan MAT-90 spectrometer. Solid-phase syntheses were performed using the Quest 210 and 205 synthesizers (Biotage, www.biotage.com). Supported liquid extractions were performed using Isolute ${ }^{\circledR}$ HM-N columns (IST, Part. No. 800-0350-EM). Sample evaporation was performed using a GeneVac ${ }^{\circledR} \mathrm{EZ}-2$ evaporator system. Analytical thin layer chromatography was performed using $0.25 \mathrm{~mm}$ silica gel $60-\mathrm{F}$ plates. Microwave reactions were carried out using the CEM Explorer/Discover (www.cem.com) system equipped with a dynamic cooling valve. Flash chromatography was performed using 200-400 mesh silica gel (Scientific Absorbent Incorporated). Methylene chloride, tetrahydrofuran, and toluene were purified by passing through two packed columns of neutral alumina (Glass Contour, Irvine, CA). Unfunctionalized polystyrene resin was obtained from Biotage/Argonaut (P/N: 800395). All other reagents and solvents were used as supplied by Sigma-Aldrich, Fluka, and Strem Chemicals. 
Representative procedures for the synthesis of silanes $(R)-4$ and $(R)-5$ :

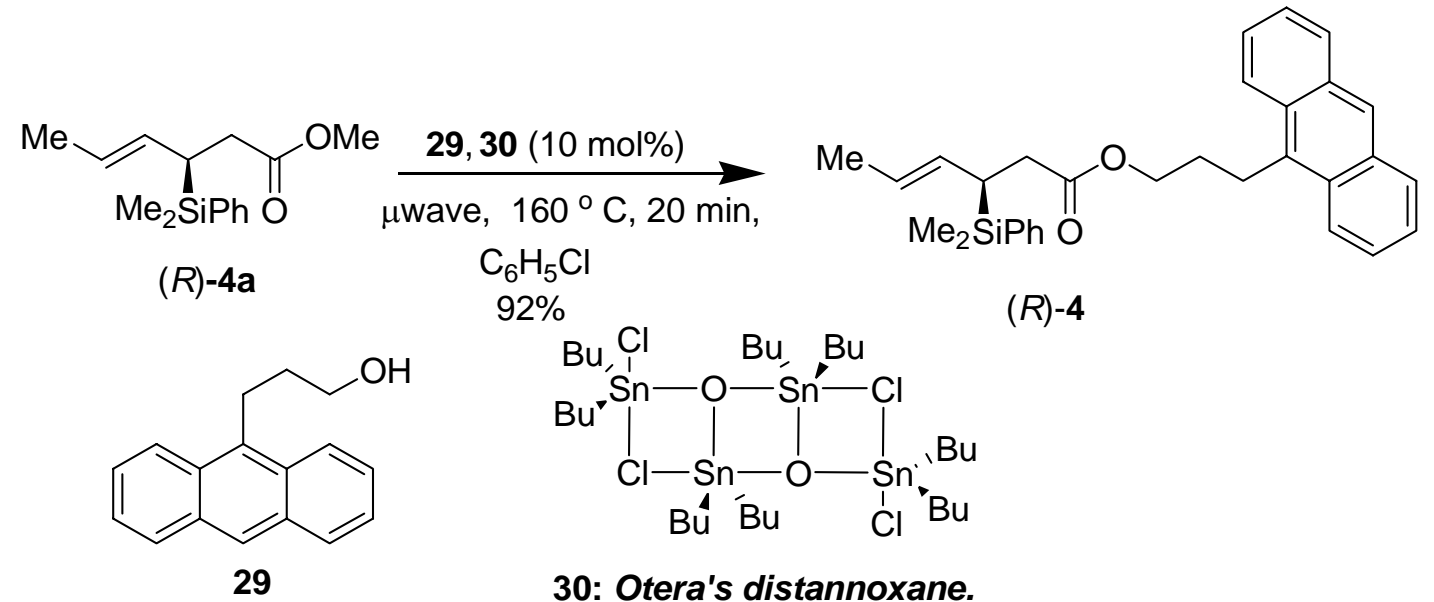

To a solution of $(R)-4 a^{\mathrm{S} 1}(1.312 \mathrm{~g}, 5.00 \mathrm{mmol})$ and alcohol $\mathbf{2 9}^{\mathrm{S} 2}(1.417 \mathrm{~g}, 6.00 \mathrm{mmol})$ in $15 \mathrm{~mL}$ of chlorobenzene was added distannoxane catalyst $30^{\mathrm{S} 3}(500 \mathrm{mg}, 0.05 \mathrm{mmol})$. The resulting mixture was irradiated in a microwave reaction vessel for 20 minutes at $160{ }^{0} \mathrm{C}(150 \mathrm{~W})$. After cooling to rt, the reaction mixture was concentrated in vacuo. The crude product was purified by flash chromatography (15:1 hexanes/EtOAc) to yield $2.14 \mathrm{~g}(4.58 \mathrm{mmol}, 92 \%)$ of $(R)-4$ as yellow oil.

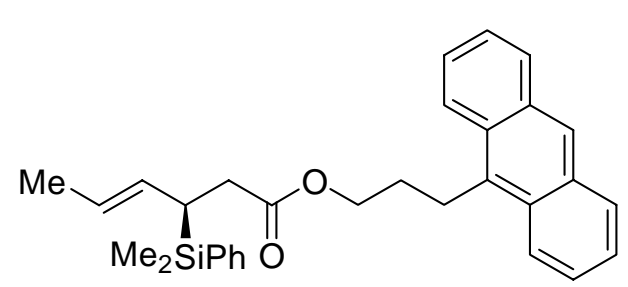

(R)-4

Tagged silane $(R)-4:{ }^{1} \mathrm{H}$ NMR $\left(400 \mathrm{MHz}, \mathrm{CDCl}_{3}\right) \delta 8.33(\mathrm{~s}$, $1 \mathrm{H}), 8.21(\mathrm{~d}, 2 \mathrm{H}, J=9.2 \mathrm{~Hz}), 7.99(\mathrm{~d}, 2 \mathrm{H}, J=9.2 \mathrm{~Hz})$, 7.52-7.30 (m, 9H), $5.31(\mathrm{~m}, 2 \mathrm{H}), 4.14(\mathrm{t}, 2 \mathrm{H}, J=6.0 \mathrm{~Hz})$, $3.63(\mathrm{t}, 2 \mathrm{H}, J=8.0 \mathrm{~Hz}), 2.42-2.23(\mathrm{~m}, 3 \mathrm{H}), 2.07(\mathrm{~m}, 2 \mathrm{H})$, $1.58(\mathrm{~d}, 3 \mathrm{H}, J=4.4 \mathrm{~Hz}), 0.30(\mathrm{~s}, 6 \mathrm{H}) ;{ }^{13} \mathrm{C} \mathrm{NMR}(100 \mathrm{MHz}$, $\left.\mathrm{CDCl}_{3}\right) \delta 173.7,137.0,134.1,133.7,131.6,130.0,129.6$, $129.3,129.2$, 127.8, 126.0, 125.6, 124.9, 124.2, 124.0, 64.1, $34.5,29.9,28.8,24.2,18.1,-0.45,-0.56:[\alpha]^{23}{ }_{\mathrm{D}}=+7.2^{\circ}\left(\mathrm{c}=0.93, \mathrm{CHCl}_{3}\right) ; \quad \mathrm{CIHRMS}[\mathrm{M}+1]^{+}$ calculated for $\mathrm{C}_{31} \mathrm{H}_{35} \mathrm{O}_{2} \mathrm{Si} 467.2397$, found 467.2393.

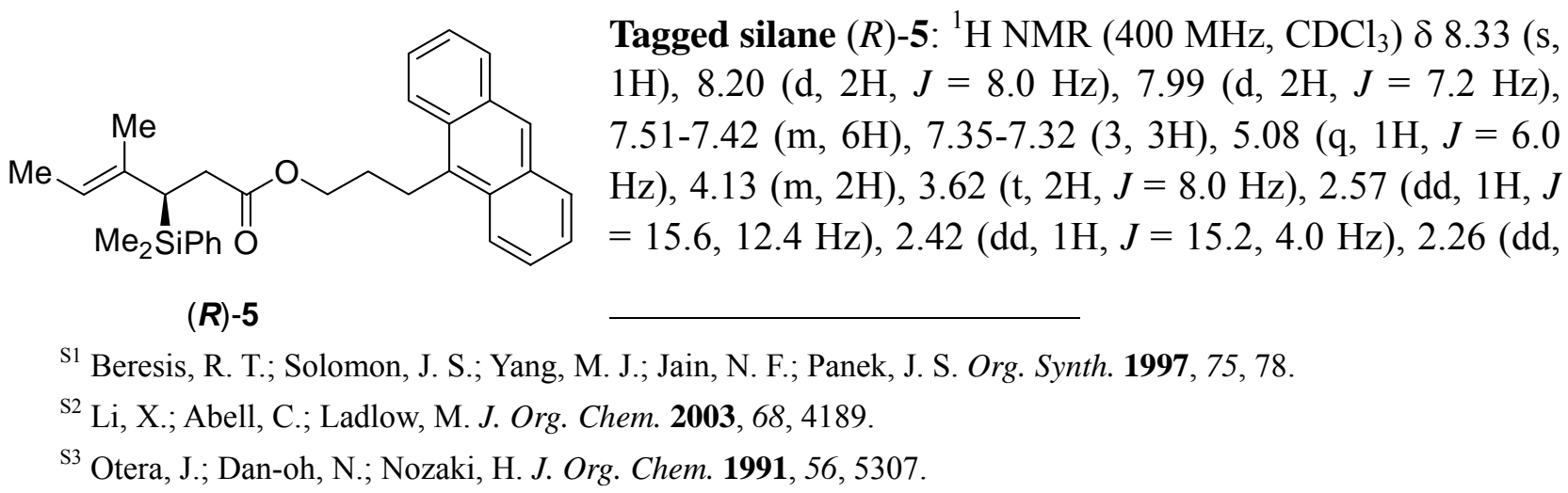


$1 \mathrm{H}, J=12.0,4.8 \mathrm{~Hz}), 2.07(\mathrm{~m}, 2 \mathrm{H}), 1.51(\mathrm{~m}, 3 \mathrm{H}), 0.33(\mathrm{~s}, 3 \mathrm{H}), 0.29(\mathrm{~s}, 3 \mathrm{H}) ;{ }^{13} \mathrm{C}$ NMR $(100$ $\left.\mathrm{MHz}, \mathrm{CDCl}_{3}\right) \delta 173.7,137.6,135.3,134.0,133.7,131.6,129.3,129.2,127.8,126.0,125.6,124.9$, $124.1,117.9,64.0,34.5,34.4,29.9,24.1,17.2,13.4,-3.8,-4.9 ;[\alpha]^{23}{ }_{\mathrm{D}}=+27.4^{\circ}\left(\mathrm{c}=0.85, \mathrm{CHCl}_{3}\right)$ CIHRMS $[\mathrm{M}+1]^{+}$calculated for $\mathrm{C}_{32} \mathrm{H}_{37} \mathrm{O}_{2} \mathrm{Si} 481.2554$, found 481.2563 .

Preparation of maleimide resin $7:^{\mathrm{S} 4}$ To a $500 \mathrm{~mL}$ 2-neck flask (pre-silanized with $5 \%$ $\mathrm{TMSCl} /$ hexanes and oven dried) were added unfunctionalized polystyrene resin $(10 \mathrm{~g})$ followed by $100 \mathrm{~mL}$ of freshly distilled $\mathrm{CH}_{2} \mathrm{Cl}_{2}$ under nitrogen. A mechanical stirring apparatus was attached and the reaction mixture was cooled to $0{ }^{\circ} \mathrm{C} . \mathrm{FeCl}_{3}(720 \mathrm{mg}, 4.52 \mathrm{mmol})$ was added followed by $N$-chloromethylmaleimide ( $2.92 \mathrm{~g}, 20 \mathrm{mmol}$ ) dissolved in $20 \mathrm{~mL}$ of $\mathrm{CH}_{2} \mathrm{Cl}_{2}$. The reaction was warmed to rt and mechanically stirred for $5 \mathrm{~h}$. The resin was filtered and washed with $2 \times 200 \mathrm{~mL}$ $\mathrm{CH}_{2} \mathrm{Cl}_{2}, 4 \times 200 \mathrm{~mL}$ 1:1 THF/aqueous $\mathrm{HCl}(1 \mathrm{M}), 4 \times 100 \mathrm{~mL}$ THF, and dried under high vacuum overnight to yield maleimide resin $7(11.8 \mathrm{~g})$. The resin was stored in the freezer under a nitrogen atmosphere.

Loading Determination for resin 7: A mixture of resin 7 (100 mg), anthracen-10-yl)propan-1-ol $29(83 \mathrm{mg}, 0.355 \mathrm{mmol})$, and 4-biphenylmethanol $(66 \mathrm{mg}, 0.355 \mathrm{mmol})$ were placed in a Quest $2105 \mathrm{~mL}$ reaction vessel and dissolved in $2.0 \mathrm{~mL}$ anhydrous DCE. The reaction was stirred for 24 $\mathrm{h}$ at $80^{\circ} \mathrm{C}$. After filtering the reaction mixture, the resin was washed with THF $(2 \times 2 \mathrm{~mL}), \mathrm{MeOH}$ $(2 \times 2 \mathrm{~mL})$, and $\mathrm{CH}_{2} \mathrm{Cl}_{2}(3 \times 2 \mathrm{~mL})$. The filtrate was collected and concentrated under reduced pressure. The resulting sample was dissolved in $10 \mathrm{~mL}$ of $\mathrm{CH}_{3} \mathrm{CN}$ and subjected to an HPLC analysis using an Agilent 1100 with diodearray detector (DAD) and an Agilent Zorbax $\mathrm{C}_{18}(3.5 \mu \mathrm{m}$, $4.6 \times 75 \mathrm{~mm}$ ) column (4-biphenylmethanol as internal standard). The loading was determined to be $1.54 \mathrm{mmol} / \mathrm{g}$ as shown below.

\begin{tabular}{|c|c|}
\hline $\begin{array}{l}\text { Area(anthracen-10-yl)propan-1-ol)/Area(internal } \\
\text { standard) }\end{array}$ & Concentration of anthracen-10-yl)propan-1-ol \\
\hline 0.01 & 0.467 \\
\hline 0.02 & 0.945 \\
\hline 0.04 & 1.245 \\
\hline
\end{tabular}

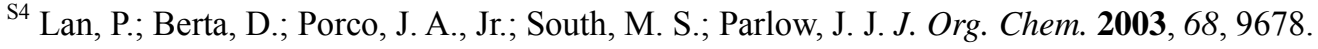




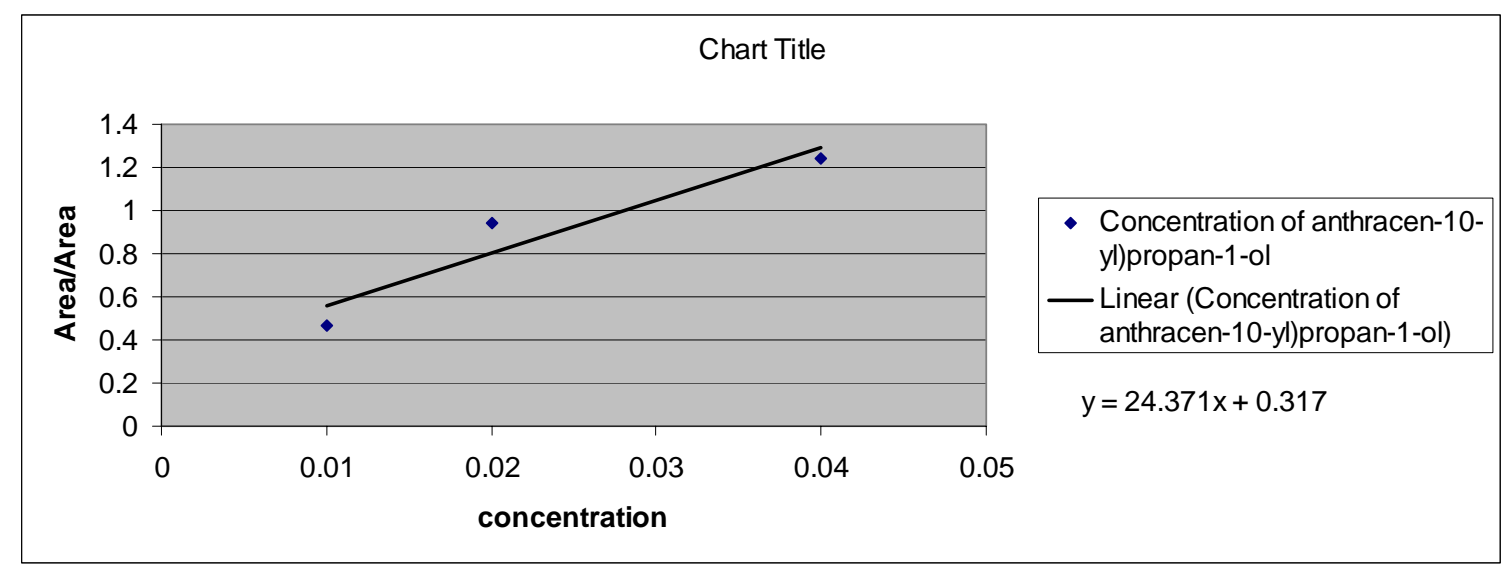

When $\mathrm{y}=0.806$ (see UV chromatogram below), $\mathrm{x}=0.0201 \mathrm{M}$

Loading $=(0.355-0.0201 \times 10 \mathrm{~mL}) / 0.100 \mathrm{~g}=1.54 \mathrm{mmol} / \mathrm{g}$

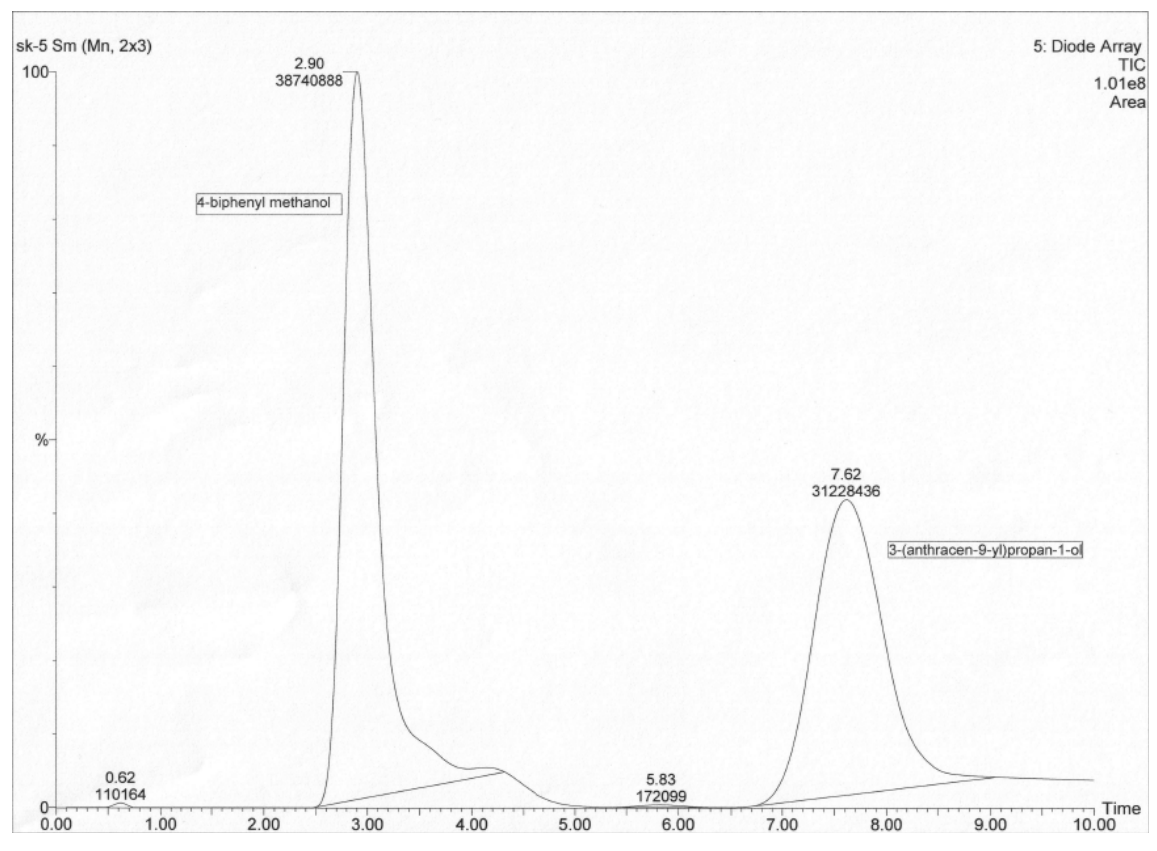<smiles>COC(OC)C(C)[C@H](OC)c1ccccc1</smiles>

9

Acetal 9: Benzaldehyde (98 mg, $0.925 \mathrm{mmol})$, TMSOMe (0.26 mL, 1.85 $\mathrm{mmol})$, and anthracene-tagged organosilane $(R)-4(520 \mathrm{mg}, 1.11 \mathrm{mmol})$ were dissolved in $\mathrm{CH}_{2} \mathrm{Cl}_{2}(6.0 \mathrm{~mL})$ and cooled to $-78{ }^{\circ} \mathrm{C}$. Triflic acid $(0.070 \mathrm{~mL}$, $0.80 \mathrm{mmol}$ ) was added to the mixture at $-78{ }^{\circ} \mathrm{C}$, and the solution was stirred at $-78{ }^{\circ} \mathrm{C}$ for $4 \mathrm{~h}$ before saturated $\mathrm{NaHCO}_{3}(4 \mathrm{~mL})$ was added. The mixture 
was extracted with $\mathrm{CH}_{2} \mathrm{Cl}_{2}(3 \times 10 \mathrm{~mL})$ and the combined organic layers were dried over $\mathrm{MgSO}_{4}$. Removal of solvent under reduced pressure afforded a pale yellow oil.

The pale yellow oil was added to a microwave reaction vessel, chlorobenzene $(10 \mathrm{~mL})$ and maleimide resin 7 (900 $\mathrm{mg}, 1.39 \mathrm{mmol}$, loading $=1.54 \mathrm{mmol} / \mathrm{g})$ were added, and the mixture was irradiated for $20 \mathrm{~min}$ at $150{ }^{\circ} \mathrm{C}(200 \mathrm{~W})$. The cooled reaction mixture was transferred to a SPE cartridge, and the resin was rinsed with $\mathrm{MeOH}(3 \times 5 \mathrm{~mL})$, THF $(5 \times 5 \mathrm{~mL}), \mathrm{CH}_{2} \mathrm{Cl}_{2}(5 \times 10 \mathrm{~mL})$ and dried to afford the resin-captured product.

After drying under a flow of nitrogen, the resin was added to $\mathrm{CH}_{2} \mathrm{Cl}_{2}(10 \mathrm{~mL}) / \mathrm{MeOH}(1 \mathrm{~mL})$, and ozone was bubbled through the solution for $20 \mathrm{~min}$ at $0{ }^{\circ} \mathrm{C}$. Dimethyl sulfide $(1.5 \mathrm{~mL})$ was added to quench the ozonide, and the reaction was allowed to stir at room temperature for $16 \mathrm{~h}$. The reaction mixture was transferred to an SPE cartridge, and was further washed with $\mathrm{CH}_{2} \mathrm{Cl}_{2}(6$ x $8 \mathrm{~mL})$. The combined liquid phase was washed with saturated $\mathrm{NaCl}$ solution $(3 \times 20 \mathrm{~mL})$ and the organic layer dried over $\mathrm{MgSO}_{4}$. Removal of solvent under reduced pressure afforded a pale yellow oil.

The crude material was combined with trimethylorthoformate $(0.51 \mathrm{~mL}, 4.63 \mathrm{mmol})$ and dissolved in $\mathrm{CH}_{2} \mathrm{Cl}_{2}(6 \mathrm{~mL})$. Montmorillonite $\mathrm{K} 10$ clay $(100 \mathrm{mg})$ was added to the mixture and solution was stirred at $\mathrm{rt}$ for $5 \mathrm{~h}$. The reaction mixture was filtered and the solvent removed under reduced pressure to afford dimethyl acetal 9 as a pale yellow oil (136 mg, 65\% yield for $4 \mathrm{steps}$, $\mathrm{dr}$ > 30:1). ${ }^{1} \mathrm{H}$ NMR $\left(400 \mathrm{MHz}, \mathrm{CDCl}_{3}\right) \delta$ 7.35-7.24 (m, 5H), $4.36(\mathrm{~d}, 1 \mathrm{H}, J=4.4 \mathrm{~Hz}), 4.16(\mathrm{~d}, 1 \mathrm{H}, J$ $=7.2 \mathrm{~Hz}), 3.38(\mathrm{~s}, 3 \mathrm{H}), 3.28(\mathrm{~s}, 3 \mathrm{H}), 3.23(\mathrm{~s}, 3 \mathrm{H}), 1.91(\mathrm{~m}, 1 \mathrm{H}), 0.84(\mathrm{~d}, 3 \mathrm{H}, J=6.8 \mathrm{~Hz}) ;{ }^{13} \mathrm{C}$ NMR $\left(75 \mathrm{MHz}, \mathrm{CDCl}_{3}\right) \delta 141.2,128.4,127.3,126.9,106.47,83.3,57.5,54.7,53.7,43.4,8.6$; IR (neat) $v_{\max }: 3085,3027,2935,2828,1453,1380,1117,1050 ;[\alpha]^{23}=-34.5^{\circ}\left(\mathrm{c}=1.00, \mathrm{CHCl}_{3}\right)$. CIHRMS $[\mathrm{M}+1]^{+}$calculated for $\mathrm{C}_{13} \mathrm{H}_{20} \mathrm{O}_{3} 225.1412$, found 225.1427.

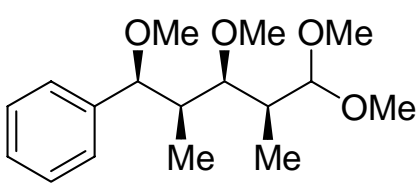

$10 a$

Acetal 10a: Dimethyl acetal 9a (85 $\mathrm{mg}, 0.380 \mathrm{mmol})$ and $(R)-4(212$ $\mathrm{mg}, 0.454 \mathrm{mmol})$ were dissolved in $\mathrm{CH}_{2} \mathrm{Cl}_{2}(3 \mathrm{~mL})$ and cooled to -78 ${ }^{\circ} \mathrm{C}$. Triflic acid $(0.033 \mathrm{~mL}, 0.378 \mathrm{mmol})$ was added to the mixture at $-78{ }^{\circ} \mathrm{C}$ and the solution was stirred at $-78{ }^{\circ} \mathrm{C}$ for $16 \mathrm{~h}$ followed by addition of saturated $\mathrm{NaHCO}_{3}(3 \mathrm{~mL})$. The mixture was extracted with $\mathrm{CH}_{2} \mathrm{Cl}_{2}(3 \times 10 \mathrm{~mL})$ and the combined organic layers were dried over $\mathrm{MgSO}_{4}$. Removal of the solvent under reduced pressure afforded a pale yellow oil. This material was added to a microwave reaction vessel, and chlorobenzene $(4 \mathrm{~mL})$ and maleimide resin 7 (400 $\mathrm{mg}, 0.570 \mathrm{mmol}$, loading $1.54 \mathrm{mmol} / \mathrm{g})$ were added and the mixture was irradiated for $20 \mathrm{~min}$ at $150{ }^{\circ} \mathrm{C}(200 \mathrm{~W})$. After cooling to $\mathrm{rt}$, the reaction mixture was transferred to an SPE cartridge, and the resin rinsed with $\mathrm{MeOH}(3 \times 5 \mathrm{~mL})$, THF $(5 \times 5 \mathrm{~mL}), \mathrm{CH}_{2} \mathrm{Cl}_{2}(5 \times 10 \mathrm{~mL})$, and dried under a flow of nitrogen to afford the resin-captured product. To the resin was added $\mathrm{CH}_{2} \mathrm{Cl}_{2}(8 \mathrm{~mL}) / \mathrm{MeOH}(0.8 \mathrm{~mL})$, and ozone was bubbled through the solution for $20 \mathrm{~min}$ at $0{ }^{\circ} \mathrm{C}$. Dimethyl sulfide $(1 \mathrm{~mL})$ was added to quench the ozonide, and the reaction was allowed to stir at $\mathrm{rt}$ for $16 \mathrm{~h}$. The reaction mixture was transferred to an SPE cartridge, and was rinsed with $\mathrm{CH}_{2} \mathrm{Cl}_{2}(5 \times 5 \mathrm{~mL})$. The combined liquid phase was washed with saturated $\mathrm{NaCl}$ solution $(3 \times 10 \mathrm{~mL})$, and the organic layer was dried over $\mathrm{MgSO}_{4}$. Removal of solvent under reduced pressure afforded a pale yellow oil. The crude material 
was combined with trimethylorthoformate $(0.207 \mathrm{~mL}, 1.89 \mathrm{mmol})$ and dissolved in $\mathrm{CH}_{2} \mathrm{Cl}_{2}(4 \mathrm{~mL})$. Montmorillonite K10 clay $(85 \mathrm{mg}$ ) was added to the mixture and the solution was stirred at $\mathrm{rt}$ for 5 $\mathrm{h}$. The reaction mixture was filtered and the solvent removed under reduced pressure to afford dimethyl acetal 10a as a pale yellow oil (72 $\mathrm{mg}, 64 \%$ yield for 4 steps, $\mathrm{dr}>20: 1)$. ${ }^{1} \mathrm{H}$ NMR (400 $\left.\mathrm{MHz}, \mathrm{CDCl}_{3}\right) \delta$ 7.35-7.20 (m, 5H), $4.27(\mathrm{~d}, 1 \mathrm{H}, J=8 \mathrm{~Hz}), 4.16(\mathrm{~d}, 1 \mathrm{H}, J=4.8 \mathrm{~Hz}), 3.37(\mathrm{~s}, 3 \mathrm{H})$, $3.35(\mathrm{~s}, 3 \mathrm{H}), 3.31(\mathrm{dd}, 1 \mathrm{H}, J=7.2,2.4 \mathrm{~Hz}), 3.28(\mathrm{~s}, 3 \mathrm{H}), 3.21(\mathrm{~s}, 3 \mathrm{H}), 2.05(\mathrm{~m}, 1 \mathrm{H}), 1.82(\mathrm{~m}, 1 \mathrm{H})$, $0.97(\mathrm{~d}, 3 \mathrm{H}, J=7.2 \mathrm{~Hz}), 0.87(\mathrm{~d}, 3 \mathrm{H}, J=7.2 \mathrm{~Hz}) ;{ }^{13} \mathrm{C} \mathrm{NMR}\left(100 \mathrm{MHz}, \mathrm{CDCl}_{3}\right) \delta 140.9,128.1$, 127.1, 126.9, 106.2, 84.3, 82.0, 60.7, 57.2, 54.0, 51.7, 43.8, 38.0, 9.8, 8.9; IR (neat) $v_{\max }: 3060$, $2936,2828,1690,1453,1379,1119,1091 ;[\alpha]^{23}=-46.5^{\circ}\left(\mathrm{c}=0.60, \mathrm{CHCl}_{3}\right) ; \mathrm{CIHRMS}[\mathrm{M}+1]^{+}$ calculated $\mathrm{C}_{17} \mathrm{H}_{28} \mathrm{O}_{4}$ 297.2067, found 297.2024.
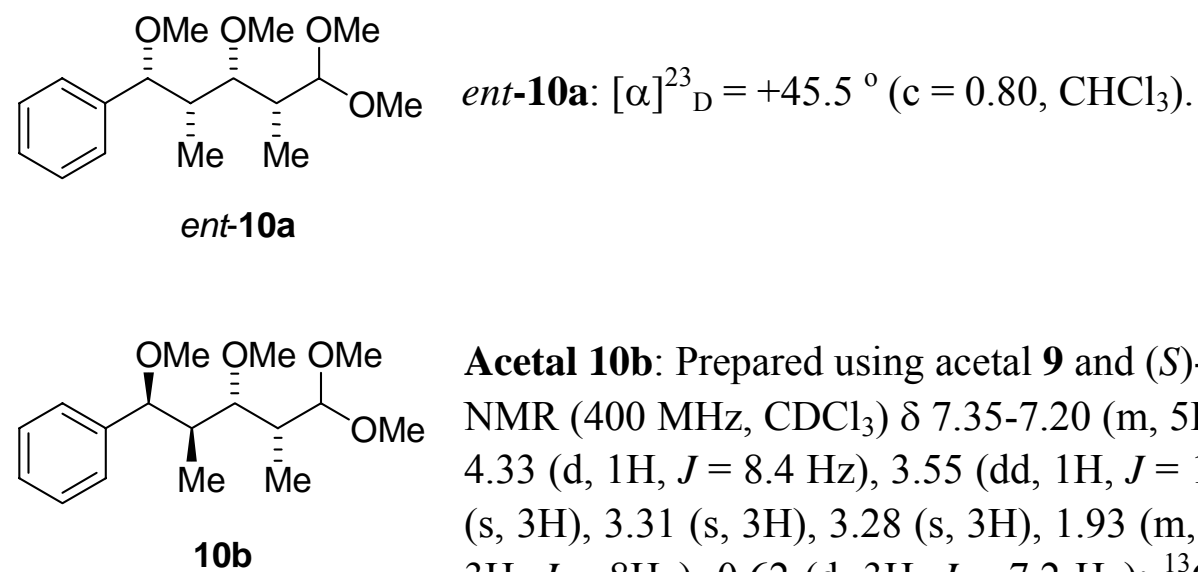

Acetal 10b: Prepared using acetal 9 and $(S)-4 ;(78 \%$ yield, $\mathrm{dr}=9: 1) .{ }^{1} \mathrm{H}$ NMR (400 MHz, $\left.\mathrm{CDCl}_{3}\right) \delta$ 7.35-7.20 (m, 5H), $4.58(\mathrm{~d}, 1 \mathrm{H}, J=1.6 \mathrm{~Hz})$, $4.33(\mathrm{~d}, 1 \mathrm{H}, J=8.4 \mathrm{~Hz}), 3.55(\mathrm{dd}, 1 \mathrm{H}, J=10,2 \mathrm{~Hz}), 3.51(\mathrm{~s}, 3 \mathrm{H}), 3.33$ $(\mathrm{s}, 3 \mathrm{H}), 3.31(\mathrm{~s}, 3 \mathrm{H}), 3.28(\mathrm{~s}, 3 \mathrm{H}), 1.93(\mathrm{~m}, 1 \mathrm{H}), 1.63(\mathrm{~m}, 1 \mathrm{H}), 0.84(\mathrm{~d}$, $3 \mathrm{H}, J=8 \mathrm{~Hz}), 0.62(\mathrm{~d}, 3 \mathrm{H}, J=7.2 \mathrm{~Hz}) ;{ }^{13} \mathrm{C} \mathrm{NMR}\left(100 \mathrm{MHz}, \mathrm{CDCl}_{3}\right)$ 129.2, 128.0, 126.7, 126.6, 105.8, 81.7, 81.6, 60.8, 56.8, 52.4, 52.0, 43.8, 37.1, 8.8, 7.9; IR (neat) $\nu_{\max }: 2976,2938,2829,1738,1690,1603,1493,1453,1381,1203,1115,1089 ;[\alpha]^{23}{ }_{\mathrm{D}}=-36.9^{\circ}(\mathrm{c}$ $\left.=0.65, \mathrm{CHCl}_{3}\right)$.<smiles>COC(OC)C(C)C(C)[C@H](C)[C@H](OC)c1ccccc1</smiles>

ent-10b

$$
\text { ent-10b:. }[\alpha]_{\mathrm{D}}^{23}=+36.8^{\circ}\left(\mathrm{c}=0.65, \mathrm{CHCl}_{3}\right) \text {. }
$$

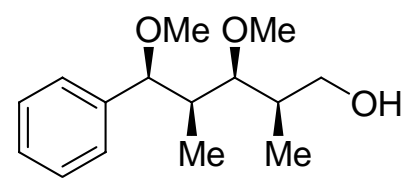

12

Alcohol 12: Dimethyl acetal 9 (85 mg, $0.380 \mathrm{mmol})$ and $(R)-4(212 \mathrm{mg}$, $0.454 \mathrm{mmol})$ were dissolved in $\mathrm{CH}_{2} \mathrm{Cl}_{2}(3 \mathrm{~mL})$ and cooled to $-78{ }^{\circ} \mathrm{C}$. Triflic acid $(0.033 \mathrm{~mL}, 0.378 \mathrm{mmol})$ was added to the mixture at $-78{ }^{\circ} \mathrm{C}$, and the solution was stirred at $-78{ }^{\circ} \mathrm{C}$ for $16 \mathrm{~h}$ before being quenched with saturated $\mathrm{NaHCO}_{3}(3 \mathrm{~mL})$. The mixture was extracted with $\mathrm{CH}_{2} \mathrm{Cl}_{2}$ $(3 \times 10 \mathrm{~mL})$ and the combined organic layers were dried over $\mathrm{MgSO}_{4}$. Removal of solvent under reduced pressure afforded a pale yellow oil.

The pale yellow oil was charged to microwave reaction vessel, chlorobenzene $(4 \mathrm{~mL})$ and 
maleimide resin $7(400 \mathrm{mg}, 0.570 \mathrm{mmol}$, loading $1.54 \mathrm{mmol} / \mathrm{g})$ were added, and the mixture irradiated for $20 \mathrm{~min}$ at $150{ }^{\circ} \mathrm{C}(200 \mathrm{~W})$. After cooling to rt, reaction mixture was transferred to an SPE cartridge, and the resin was rinsed with $\mathrm{MeOH}(3 \times 5 \mathrm{~mL})$, THF $(5 \times 5 \mathrm{~mL}), \mathrm{CH}_{2} \mathrm{Cl}_{2}(5 \times 10$ $\mathrm{mL}$ ) and dried under a flow of nitrogen to afford the resin-captured product. To the resin was added $\mathrm{CH}_{2} \mathrm{Cl}_{2}(6 \mathrm{~mL}) / \mathrm{MeOH}(3 \mathrm{~mL})$ and ozone was bubbled through the solution for $20 \mathrm{~min}$ at 0 ${ }^{\circ} \mathrm{C}$. Sodium borohydride $(100 \mathrm{mg})$ was added and the reaction was allowed to stir at $\mathrm{rt}$ for 16 hours. The reaction mixture was transferred to an SPE cartridge and was rinsed with $0.5 \mathrm{~N} \mathrm{HCl}(2 \times 2$ $\mathrm{mL})$, THF $(2 \times 5 \mathrm{~mL}) \mathrm{CH}_{2} \mathrm{Cl}_{2}(5 \times 5 \mathrm{~mL})$. The combined liquid phase was extracted with saturated aqueous $\mathrm{NaCl}(3 \times 10 \mathrm{~mL})$, and the organic layer was dried over $\mathrm{MgSO}_{4}$. Removal of the solvent under reduced pressure afforded $12(60 \mathrm{mg}, 62 \%$ yield, $\mathrm{dr}=20: 1)$.

${ }^{1} \mathrm{H}$ NMR $\left(400 \mathrm{MHz}, \mathrm{CDCl}_{3}\right) \delta$ 7.37-7.23 (m, 5H), $4.13(\mathrm{~d}, 1 \mathrm{H}, J=5.6 \mathrm{~Hz}), 3.65(\mathrm{dd}, 1 \mathrm{H}, J=7.2$, $6.8 \mathrm{~Hz}), 3.54(\mathrm{dd}, J=6.8 \mathrm{~Hz}, 4.8 \mathrm{~Hz}), 3.35(\mathrm{~s}, 3 \mathrm{H}), 3.26(\mathrm{~s}, 3 \mathrm{H}), 3.14(\mathrm{t}, 1 \mathrm{H}, J=5.2 \mathrm{~Hz}), 1.93-2.00$ $(\mathrm{m}, 2 \mathrm{H}), 0.99(\mathrm{~d}, 3 \mathrm{H}, J=6.4 \mathrm{~Hz}), 0.91(\mathrm{~d}, 3 \mathrm{H}, J=7.2 \mathrm{~Hz}) ;{ }^{13} \mathrm{C} \mathrm{NMR}\left(100 \mathrm{MHz}, \mathrm{CDCl}_{3}\right) \delta 144.0$, 128.5, 127.6, 127.4, 85.6, 84.2, 66.7, 60.3, 57.4, 42.7, 37.9, 11.8, 10.5; IR (neat) $v_{\max }: 3420,2970$, 2927, 1456, 1087, 730; $[\alpha]^{23}{ }_{\mathrm{D}}=-14.6^{\circ}\left(\mathrm{c}=0.2, \mathrm{CHCl}_{3}\right) ; \mathrm{CIHRMS}[\mathrm{M}+1]^{+}$calculated $\mathrm{C}_{15} \mathrm{H}_{24} \mathrm{O}_{3}$ 253.1725 , found 253.1828 .

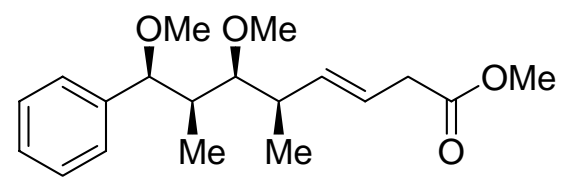

13

Ester 13: Dimethyl acetal 9 (42 $\mathrm{mg}, 0.190 \mathrm{mmol})$ and $(R)-4(90$ $\mathrm{mg}, 0.188 \mathrm{mmol})$ were dissolved in $\mathrm{CH}_{2} \mathrm{Cl}_{2}(1 \mathrm{~mL})$ and cooled to $-78{ }^{\circ} \mathrm{C}$. Triflic acid $(16 \mu \mathrm{L}, 0.180 \mathrm{mmol})$ was added to the mixture at $-78{ }^{\circ} \mathrm{C}$, and the solution was stirred at $-78{ }^{\circ} \mathrm{C}$ for 16 $\mathrm{h}$ and quenched by addition of saturated $\mathrm{NaHCO}_{3}(1 \mathrm{~mL})$. The mixture was extracted with $\mathrm{CH}_{2} \mathrm{Cl}_{2}(3 \times 3 \mathrm{~mL})$ and the combined organic layers were dried over $\mathrm{MgSO}_{4}$. Removal of solvent under reduced pressure afforded an oil.

The pale yellow oil was charged to microwave reaction vessel, chlorobenzene $(3 \mathrm{~mL})$ and maleimide resin 7 (180 $\mathrm{mg}, 0.270 \mathrm{mmol}$, loading $1.54 \mathrm{mmol} / \mathrm{g}$ ) were added, and the mixture was irradiated for $20 \mathrm{~min}$ at $150{ }^{\circ} \mathrm{C}(200 \mathrm{~W})$. The cooled reaction mixture was transferred to an SPE cartridge and the resin was rinsed with $\mathrm{MeOH}(3 \times 2 \mathrm{~mL})$, THF $(5 \times 1 \mathrm{~mL}), \mathrm{CH}_{2} \mathrm{Cl}_{2}(5 \times 3 \mathrm{~mL})$ and dried under a flow of nitrogen to yield the resin-captured product. To the resin was added THF (2 $\mathrm{mL}) / \mathrm{MeOH}(0.5 \mathrm{~mL})$. Freshly prepared $1 \mathrm{M} \mathrm{NaOMe}(0.3 \mathrm{~mL}, 0.3 \mathrm{mmol})$ was added and the reaction mixture was agitated for $2 \mathrm{~h}$. The reaction mixture was transferred to an SPE cartridge, and was rinsed with $0.5 \mathrm{~N} \mathrm{HCl}(2 \times 1 \mathrm{~mL})$, THF $(2 \times 2 \mathrm{~mL}) \mathrm{CH}_{2} \mathrm{Cl}_{2}(5 \times 2 \mathrm{~mL})$. The combined organic phase was washed with saturated aqueous $\mathrm{NaCl}(3 \times 5 \mathrm{~mL})$, and the organic layer dried over $\mathrm{MgSO}_{4}$. Removal of the solvent under reduced pressure afforded ester $14 \mathrm{a}(41 \mathrm{mg}, 69 \%$ yield, $\mathrm{dr}=20: 1) .{ }^{1} \mathrm{H}$ NMR $\left(400 \mathrm{MHz}, \mathrm{CDCl}_{3}\right) \delta$ 7.32-7.27 (m, 5H) $5.51(\mathrm{dt}, 1 \mathrm{H}, J=10.8 \mathrm{~Hz}, 6.8 \mathrm{~Hz})$, $5.38(\mathrm{dd}, 1 \mathrm{H}, J=10.8 \mathrm{~Hz}, 7.2 \mathrm{~Hz}), 4.06(\mathrm{~d}, 1 \mathrm{H}, J=7.6 \mathrm{~Hz}), 3.70(\mathrm{~s}, 3 \mathrm{H}), 3.31(\mathrm{~s}, 3 \mathrm{H}), 3.18(\mathrm{~s}, 3 \mathrm{H})$, $3.04(\mathrm{~d}, 2 \mathrm{H}, J=6.8 \mathrm{~Hz}), 2.68-2.71(\mathrm{~m}, 1 \mathrm{H}), 2.43-249(\mathrm{~m}, 1 \mathrm{H}), 1.99-2.03(\mathrm{~m}, 1 \mathrm{H}), 1.01(\mathrm{~d}, 3 \mathrm{H}, J=$ $6.4 \mathrm{~Hz}), 0.97(\mathrm{~d}, 3 \mathrm{H}, J=6.4 \mathrm{~Hz}) ;{ }^{13} \mathrm{C} \mathrm{NMR}\left(100 \mathrm{MHz}, \mathrm{CDCl}_{3}\right) \delta 172.5,141.2,137.7,129.2$, 128.3, 127.8, 121.4, 86.6, 85.6, 60.5, 57.1, 52.0, 42.5, 40.0, 38.3, 16.3, 10.2; IR (neat) $v_{\max }: 3060$, $2974,2934,1741,1453,1092,703 ;[\alpha]^{23}=-21.3^{\circ}\left(\mathrm{c}=0.4, \mathrm{CHCl}_{3}\right) ; \mathrm{CIHRMS}[\mathrm{M}]^{+}$calculated $\mathrm{C}_{19} \mathrm{H}_{28} \mathrm{O}_{4} 320.1988$, found 320.1980 . 


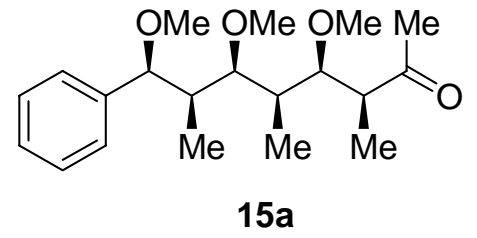

Ketone 15a: Dimethyl acetal 10a (22 $\mathrm{mg}, 0.074 \mathrm{mmol})$ and $(R)-5$ (39 $\mathrm{mg}, 0.081 \mathrm{mmol})$ were dissolved in $\mathrm{CH}_{2} \mathrm{Cl}_{2}(1 \mathrm{~mL})$ and cooled to -78 ${ }^{\circ} \mathrm{C}$. Boron trifluoride etherate $(0.019 \mathrm{~mL}, 0.148 \mathrm{mmol})$ was added to the mixture and the reaction mixture was warmed to $-10{ }^{\circ} \mathrm{C}$. The reaction was stirred at $-10{ }^{\circ} \mathrm{C}$ for $16 \mathrm{~h}$ and quenched by addition of saturated aqueous $\mathrm{NaHCO}_{3}(1 \mathrm{~mL})$. The mixture was extracted with $\mathrm{CH}_{2} \mathrm{Cl}_{2}(3 \times 3 \mathrm{~mL})$ and the combined organic layers were dried over $\mathrm{MgSO}_{4}$. Removal of solvent under reduced pressure afforded a pale yellow oil. The pale yellow oil was charged to microwave reaction vessel, chlorobenzene $(2 \mathrm{~mL})$ and maleimide resin $7(120 \mathrm{mg}, 0.18 \mathrm{mmol}$, loading $1.54 \mathrm{mmol} / \mathrm{g})$ were added and the mixture was irradiated for $20 \mathrm{~min}$ at $150{ }^{\circ} \mathrm{C}(200 \mathrm{~W})$. After cooling to rt, the mixture was transferred to an SPE cartridge, and the resin was rinsed with $\mathrm{CH}_{2} \mathrm{Cl}_{2}(5 \times 3 \mathrm{~mL})$ and dried under a flow of nitrogen to afford the resin-captured product. To the resin was added $\mathrm{CH}_{2} \mathrm{Cl}_{2}(2 \mathrm{~mL})$ $/ \mathrm{MeOH}(0.2 \mathrm{~mL})$ and ozone was bubbled through the solution for $20 \mathrm{~min}$ at $0{ }^{\circ} \mathrm{C}$. Dimethyl sulfide $(0.75 \mathrm{~mL})$ was added to quench the ozonide and the reaction was allowed to stir at $\mathrm{rt}$ for $16 \mathrm{~h}$. The reaction mixture was transferred to an SPE cartridge and was rinsed with $\mathrm{CH}_{2} \mathrm{Cl}_{2}(5 \times 3 \mathrm{~mL})$. The combined liquid phase was washed with saturated aqueous $\mathrm{NaCl}(3 \times 5 \mathrm{~mL})$, and the organic layer dried over $\mathrm{MgSO}_{4}$. Removal of solvent under reduced pressure afforded methyl ketone 15a as a pale yellow oil $(13 \mathrm{mg}, 52 \%$ for $3 \mathrm{steps}, \mathrm{dr}=10: 1) .{ }^{1} \mathrm{H} \mathrm{NMR}\left(400 \mathrm{MHz}, \mathrm{CDCl}_{3}\right) \delta$ 7.37-7.24 (m, $5 \mathrm{H}), 4.08(\mathrm{~d}, 1 \mathrm{H}, J=6 \mathrm{~Hz}), 3.37(\mathrm{~s}, 3 \mathrm{H}), 3.35(\mathrm{ovrlp}, 1 \mathrm{H}), 3.17(\mathrm{~s}, 3 \mathrm{H}), 3.16(\mathrm{~s}, 3 \mathrm{H}), 2.92(\mathrm{dd}, 1 \mathrm{H}$, $J=5.2,4.4 \mathrm{~Hz}), 2.70(\mathrm{~m}, 1 \mathrm{H}), 2.17(\mathrm{~s}, 3 \mathrm{H}), 1.93(\mathrm{~m}, 1 \mathrm{H}), 1.77(\mathrm{~m}, 1 \mathrm{H}), 1.06(\mathrm{~d}, 3 \mathrm{H}, J=7.2 \mathrm{~Hz})$, $0.95(\mathrm{~d}, 3 \mathrm{H}, J=6.4 \mathrm{~Hz}), 0.94(\mathrm{~d}, 3 \mathrm{H}, J=7.6 \mathrm{~Hz}) ;{ }^{13} \mathrm{C} \mathrm{NMR}\left(75 \mathrm{MHz}, \mathrm{CDCl}_{3}\right) \delta 211.3,141.0,128$. $3,127.5,127.4,85.5,83.4,82.7,60.6,60.3,57.0,49.5,43.9,40.1,29.7,29.3,11.3,10.0,9.8$; IR (neat) $v_{\max }$ : 2927, 1712,1456,1090; $[\alpha]^{23}=-26.7^{\circ}\left(\mathrm{c}=0.30, \mathrm{CHCl}_{3}\right)$; CIHRMS $[\mathrm{M}+1]^{+}$ calculated for $\mathrm{C}_{20} \mathrm{H}_{32} \mathrm{O}_{4} 337.2380$, found 337.2371.
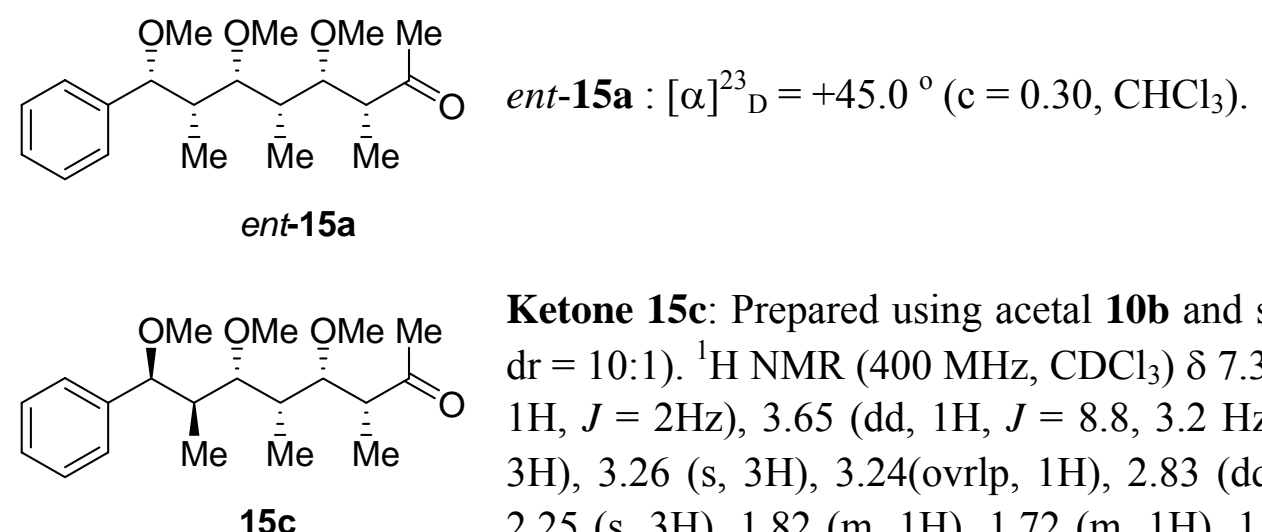

Ketone 15c: Prepared using acetal 10b and silane $(S)-5 ;(60 \%$ yield, $\mathrm{dr}=10: 1) .{ }^{1} \mathrm{H}$ NMR $\left(400 \mathrm{MHz}, \mathrm{CDCl}_{3}\right) \delta$ 7.35-7.19 (m, 5H), $4.56(\mathrm{~d}$, $1 \mathrm{H}, J=2 \mathrm{~Hz}), 3.65(\mathrm{dd}, 1 \mathrm{H}, J=8.8,3.2 \mathrm{~Hz}), 3.56(\mathrm{~s}, 3 \mathrm{H}), 3.34(\mathrm{~s}$, $3 \mathrm{H}), 3.26(\mathrm{~s}, 3 \mathrm{H}), 3.24(\mathrm{ovrlp}, 1 \mathrm{H}), 2.83(\mathrm{dq}, 1 \mathrm{H}, J=6.8,2.8 \mathrm{~Hz})$, $2.25(\mathrm{~s}, 3 \mathrm{H}), 1.82(\mathrm{~m}, 1 \mathrm{H}), 1.72(\mathrm{~m}, 1 \mathrm{H}), 1.11(\mathrm{~d}, 1 \mathrm{H}, J=7.2 \mathrm{~Hz})$, 0.90 (d, $1 \mathrm{H}, J=6.4 \mathrm{~Hz}), 0.64(\mathrm{~d}, 1 \mathrm{H}, J=7.2 \mathrm{~Hz}),{ }^{13} \mathrm{C}$ NMR $\left(75 \mathrm{MHz}, \mathrm{CDCl}_{3}\right) 212.2,141.3,128.2$, $126.9,126.8,83.9,83.0,81.8,60.7,60.4,56.7,48.9,43.9,38.6,29.0,9.8,9.1$; IR (neat) $v_{\max }: 2974$, $1927,1712,1603,1493,1454,1380,1088,705 ;[\alpha]^{23}=-28.8^{\circ}\left(\mathrm{c}=0.60, \mathrm{CHCl}_{3}\right) ; \mathrm{CIHRMS}$ $[\mathrm{M}+1]^{+}$calculated for $\mathrm{C}_{20} \mathrm{H}_{32} \mathrm{O}_{4} 337.2380$, found 337.2345. 


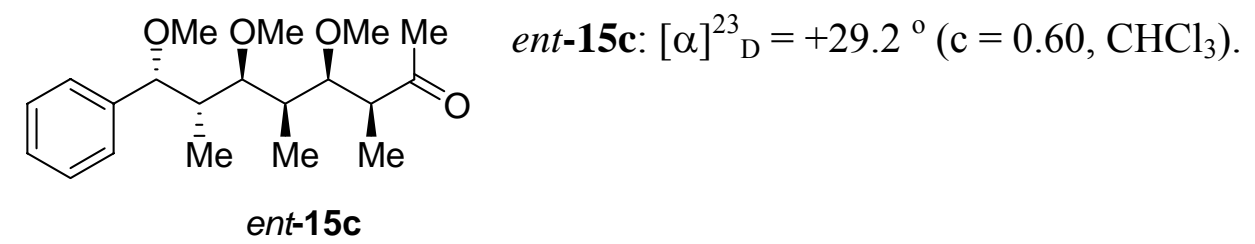

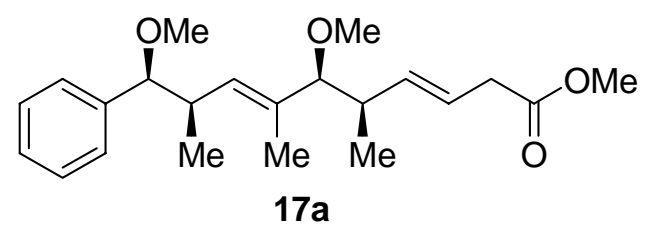

$17 a$

Ester 17a: Dimethyl acetal 10a (22 $\mathrm{mg}, 0.074 \mathrm{mmol})$ and (R)-4 (30 mg, $0.073 \mathrm{mmol})$ were dissolved in $\mathrm{CH}_{2} \mathrm{Cl}_{2}(1$ $\mathrm{mL})$ and cooled to $-78{ }^{\circ} \mathrm{C}$. Triflic acid $(10 \mu \mathrm{L}, 0.091 \mathrm{mmol})$ was added to the mixture at $-78{ }^{\circ} \mathrm{C}$, and the solution was stirred at $-78{ }^{\circ} \mathrm{C}$ for $16 \mathrm{~h}$ before being quenched with saturated aqueous $\mathrm{NaHCO}_{3}(1 \mathrm{~mL})$. The mixture was extracted with $\mathrm{CH}_{2} \mathrm{Cl}_{2}(3 \times 3 \mathrm{~mL})$ and the combined organic layers were dried over $\mathrm{MgSO}_{4}$. Removal of the solvent under reduced pressure afforded the crude crotylation product as an oil.

The pale yellow oil was charged to microwave reaction vessel, chlorobenzene $(2 \mathrm{~mL})$ and maleimide resin 7 (100 $\mathrm{mg}, 0.15 \mathrm{mmol}$, loading $1.54 \mathrm{mmol} / \mathrm{g})$ were added and the mixture was irradiated for $20 \mathrm{~min}$ at $150{ }^{\circ} \mathrm{C}(200 \mathrm{~W})$. After cooling to $\mathrm{rt}$, the reaction mixture was transferred to an SPE cartridge. The resin was rinsed with $\mathrm{MeOH}(3 \times 2 \mathrm{~mL})$, THF $(5 \times 1 \mathrm{~mL})$, and $\mathrm{CH}_{2} \mathrm{Cl}_{2}(5 \times 3$ $\mathrm{mL}$ ) and dried under a flow of nitrogen to afford the resin-captured product. To the resin was added THF $(2 \mathrm{~mL}) / \mathrm{MeOH}(0.5 \mathrm{~mL})$ followed by freshly prepared $1 \mathrm{M} \mathrm{NaOMe}(0.3 \mathrm{~mL}, 0.3$ mmol) and the reaction mixture was agitated for $2 \mathrm{~h}$. The mixture was transferred to an SPE cartridge, and was rinsed with $0.5 \mathrm{~N} \mathrm{HCl}(2 \times 1 \mathrm{~mL})$, THF $(2 \times 2 \mathrm{~mL}) \mathrm{CH}_{2} \mathrm{Cl}_{2}(5 \times 2 \mathrm{~mL})$. The combined liquid phase was washed with saturated $\mathrm{NaCl}$ solution $(3 \times 5 \mathrm{~mL})$, and the organic layer was dried over $\mathrm{MgSO}_{4}$. Removal of solvent under reduced pressure afforded compound 17a as a pale yellow oil (16 mg, 60\% yield, dr $>20: 1)$; ${ }^{1} \mathrm{H}$ NMR $\left(400 \mathrm{MHz}, \mathrm{CDCl}_{3}\right) \delta$ 7.32-7.27 (m, 5H), $5.52(\mathrm{dt}, 1 \mathrm{H}, J=16 \mathrm{~Hz}, J=6.8 \mathrm{~Hz}), 5.23(\mathrm{dd}, 1 \mathrm{H}, J=16 \mathrm{~Hz}, J=7.2 \mathrm{~Hz}), 5.14(\mathrm{~d}, 1 \mathrm{H}, J=9.6 \mathrm{~Hz})$, $3.87(\mathrm{~d}, 1 \mathrm{H}, J=6.8 \mathrm{~Hz}), 3.66(\mathrm{~s}, 3 \mathrm{H}), 3.18(\mathrm{~s}, 3 \mathrm{H}), 3.19$ (par obsc, $1 \mathrm{H}), 3.04(\mathrm{~s}, 3 \mathrm{H}), 3.01$ (d, $2 \mathrm{H} J$ $=6.8 \mathrm{~Hz}), 2.82-2.76(\mathrm{~m}, 1 \mathrm{H}), 2.26-2.16(\mathrm{~m}, 1 \mathrm{H}), 1.48(\mathrm{~s}, 3 \mathrm{H}), 1.26(\mathrm{~d}, 3 \mathrm{H}, J=6.8 \mathrm{~Hz}), 0.96(\mathrm{~d}, 3 \mathrm{H}$, $J=6.8 \mathrm{~Hz}) ;{ }^{13} \mathrm{C} \mathrm{NMR}\left(100 \mathrm{MHz}, \mathrm{CDCl}_{3}\right) \delta 172.7,141.1,136.7,132.8,132.2,128.3,127.7,127.6$, $120.9,91.3,88.3,57.2,56.2,51.9,39.8,39.3,38.2,17.2,16.5,12.1$; IR (neat) $v_{\max }: 3060,2927$, 2828, 1740, 1558, 1456, 1091; $[\alpha]^{23}{ }_{\mathrm{D}}=+31.2^{\circ}\left(\mathrm{c}=0.40, \mathrm{CHCl}_{3}\right)$; CIHRMS $[\mathrm{M}+1]^{+}$calculated for $\mathrm{C}_{22} \mathrm{H}_{32} \mathrm{O}_{4} 361.2301$, found 361.2341.

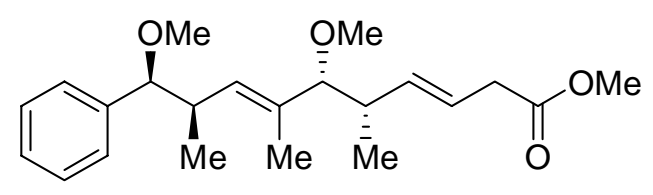

$17 \mathrm{~b}$

Ester 17b: Prepared using dimethyl acetal 10a and silane (S)-4; (59\% yield, dr > 20:1).

${ }^{1} \mathrm{H}$ NMR (400 MHz, $\left.\mathrm{CDCl}_{3}\right) \delta$ 7.32-7.27 (m, 5H), $5.42(\mathrm{dt}$, $1 \mathrm{H}, J=16 \mathrm{~Hz}, J=6.4 \mathrm{~Hz}), 5.23(\mathrm{dd}, 1 \mathrm{H}, J=16 \mathrm{~Hz}, J=$ $7.2 \mathrm{~Hz}), 4.98(\mathrm{~d}, 1 \mathrm{H}, J=9.2 \mathrm{~Hz}), 3.82(\mathrm{~d}, 1 \mathrm{H} J=6.8 \mathrm{~Hz})$, $3.62(\mathrm{~s}, 3 \mathrm{H}), 3.20(\mathrm{~s}, 3 \mathrm{H}), 2.95(\mathrm{~d}, 2 \mathrm{H}, J=6.8 \mathrm{~Hz}), 2.87(\mathrm{~d}, 1 \mathrm{H}, J=6.8 \mathrm{~Hz}), 2.75-2.79(\mathrm{~m}, 1 \mathrm{H})$, $2.84(\mathrm{~s}, 3 \mathrm{H}), 2.26-2.16(\mathrm{~m}, 1 \mathrm{H}), 1.32(\mathrm{~s}, 3 \mathrm{H}), 1.06(\mathrm{~d}, 3 \mathrm{H}, J=6.8 \mathrm{~Hz}), 0.96(\mathrm{~d}, 3 \mathrm{H}, J=6.8 \mathrm{~Hz})$; ${ }^{13} \mathrm{C} \mathrm{NMR}\left(100 \mathrm{MHz}, \mathrm{CDCl}_{3}\right) \delta 172.5,141.4,136.8,133.2,132.4,128.2,127.6,100.7,91.8,88.7$, 
$57.2,55.7,51.9,39.9,39.7,38.1,29.9,12.6,11.3,11.0 ; v_{\max }: 3060,2931,2828,1741,1558,1456$, $1091 ;[\alpha]^{23}{ }_{D}=+21.5^{\circ}\left(\mathrm{c}=0.10, \mathrm{CHCl}_{3}\right)$.

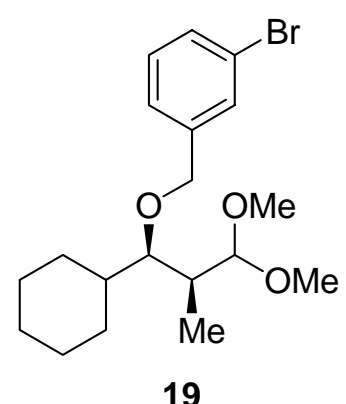

19

Acetal 19: Cyclohexanecarboxaldehyde (115 $\mathrm{mg}, \quad 0.990 \quad \mathrm{mmol})$, 3-bromobenzyloxytrimethylsilane $(522 \mathrm{mg}, 1.99 \mathrm{mmol})$ and organosilane (R)-4 $(520 \mathrm{mg}, 1.11 \mathrm{mmol})$ were dissolved in $\mathrm{CH}_{2} \mathrm{Cl}_{2}(6.0 \mathrm{~mL})$ and cooled to $-78{ }^{\circ} \mathrm{C}$. Triflic acid $(0.087 \mathrm{~mL}, 1.00 \mathrm{mmol})$ was added to the mixture at -78 ${ }^{\circ} \mathrm{C}$, and the solution was warmed to $-60{ }^{\circ} \mathrm{C}$ and stirred at this temperature for $24 \mathrm{~h}$ before being quenched by addition of saturated aqueous $\mathrm{NaHCO}_{3}(4$ $\mathrm{mL})$. The mixture was extracted with $\mathrm{CH}_{2} \mathrm{Cl}_{2}(3 \times 10 \mathrm{~mL})$ and the combined organic layers were dried over $\mathrm{MgSO}_{4}$. Removal of solvent under reduced pressure afforded the crotylation product as a yellow oil.

The pale yellow oil was added to a microwave reaction vessel, chlorobenzene $(4 \mathrm{~mL})$ and maleimide resin $7(900 \mathrm{mg}, 1.40 \mathrm{mmol}$, loading $1.54 \mathrm{mmol} / \mathrm{g}$ ) were added, and the mixture irradiated for $20 \mathrm{~min}$ at $150{ }^{\circ} \mathrm{C}(200 \mathrm{~W})$. After cooling to rt, mixture was transferred to an SPE cartridge. The resin was rinsed with $\mathrm{MeOH}(3 \times 5 \mathrm{~mL})$, THF $(5 \times 5 \mathrm{~mL})$, and $\mathrm{CH}_{2} \mathrm{Cl}_{2}(5 \times 10 \mathrm{~mL})$ and dried under a flow of nitrogen to afford the resin-captured product. To the resin was added $\mathrm{CH}_{2} \mathrm{Cl}_{2}(10 \mathrm{~mL}) / \mathrm{MeOH}(1 \mathrm{~mL})$, and ozone was bubbled through the solution for $20 \mathrm{~min}$ at $0{ }^{\circ} \mathrm{C}$. Excess dimethyl sulfide $(1.5 \mathrm{~mL})$ was added to quench the ozonide, and the reaction was allowed to stir at $\mathrm{rt}$ for $16 \mathrm{~h}$. The reaction mixture was transferred to an SPE cartridge, and was washed with $\mathrm{CH}_{2} \mathrm{Cl}_{2}(6 \times 8 \mathrm{~mL})$. The combined liquid phase was washed with saturated $\mathrm{NaCl}$ solution $(3 \mathrm{x}$ $20 \mathrm{~mL}$ ), and the organic layer was dried over $\mathrm{MgSO}_{4}$. Removal of solvent under reduced pressure afforded a pale yellow oil. The crude material was combined with trimethylorthoformate $(0.51 \mathrm{~mL}$, $4.63 \mathrm{mmol})$ and dissolved in $\mathrm{CH}_{2} \mathrm{Cl}_{2}(6 \mathrm{~mL})$. Montmorillonite $\mathrm{K} 10$ clay $(100 \mathrm{mg})$ was added to the mixture and the solution was stirred at $\mathrm{rt}$ for $5 \mathrm{~h}$. The reaction mixture was filtered and the solvent removed under reduced pressure to afford dimethyl acetal $19(225 \mathrm{mg}, 59 \%$ yield, $\mathrm{dr}>20: 1) ;{ }^{1} \mathrm{H}$ NMR (400 MHz, $\left.\mathrm{CDCl}_{3}\right) \delta 7.48(\mathrm{~s}, 1 \mathrm{H}), 7.38(\mathrm{~d}, 1 \mathrm{H}, J=7.6 \mathrm{~Hz}), 7.27-7.13(\mathrm{~m}, 2 \mathrm{H}), 4.27(\mathrm{~d}, 1 \mathrm{H}$, $J=8.4 \mathrm{~Hz}$ ), 3.36-3.31 (1H, ovrlp), 3.31 (s, $3 \mathrm{H}), 3.28(\mathrm{~s}, 3 \mathrm{H}), 2.03-2.00(\mathrm{~m}, 2 \mathrm{H}), 1.75-1.54(\mathrm{~m}, 6 \mathrm{H})$, 1.26-0.99 (m, 4H), $0.93(\mathrm{~d}, 3 \mathrm{H}, J=6.8 \mathrm{~Hz}) ;{ }^{13} \mathrm{C} \mathrm{NMR}\left(100 \mathrm{MHz}, \mathrm{CDCl}_{3}\right) \delta 141.9,130.4,130.3$, 130.1, 125.8, 122.6, 106.1, 84.0, 74.0, 53.0, 52.6, 41.2, 37.0, 30.3, 29.7, 26.7, 26.3, 8.9; IR (neat) $v_{\max }: 2925,2850,1520,1507,1472,1110,952 ;[\alpha]^{23}{ }_{\mathrm{D}}=+34.2^{\circ}\left(\mathrm{c}=0.2, \mathrm{CHCl}_{3}\right)$.

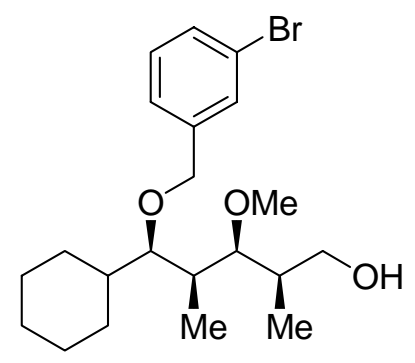

20
Alcohol 20: Acetal $19(40 \mathrm{mg}, 0.104 \mathrm{mmol})$ and $(R)-4(123 \mathrm{mg}, 0.30$ mmol) were dissolved in $\mathrm{CH}_{2} \mathrm{Cl}_{2}(1 \mathrm{~mL})$ and cooled to $-60{ }^{\circ} \mathrm{C}$. Triflic acid $(12 \mu \mathrm{L}, 0.101 \mathrm{mmol}))$ was added to the mixture at $-60{ }^{\circ} \mathrm{C}$, and the solution was stirred at $-78{ }^{\circ} \mathrm{C}$ for $24 \mathrm{~h}$ and quenched with saturated aqueous $\mathrm{NaHCO}_{3}(3 \mathrm{~mL})$. The mixture was extracted with $\mathrm{CH}_{2} \mathrm{Cl}_{2}(3 \mathrm{x}$ $10 \mathrm{~mL}$ ) and the combined organic layers were dried over $\mathrm{MgSO}_{4}$. Removal of solvent under reduced pressure afforded a yellow oil.

The pale yellow oil was added to a microwave reaction vessel, 
chlorobenzene $(4 \mathrm{~mL})$ and maleimide resin $7(400 \mathrm{mg}, 0.450 \mathrm{mmol}$, loading $1.54 \mathrm{mmol} / \mathrm{g}$ ) were added and the mixture was irradiated for $20 \mathrm{~min}$ at $150{ }^{\circ} \mathrm{C}(200 \mathrm{~W})$. The cooled reaction mixture was transferred to a cartridge, and the solid phase was rinsed with $\mathrm{MeOH}(3 \times 5 \mathrm{~mL})$, THF $(5 \times 5$ $\mathrm{mL})$, and $\mathrm{CH}_{2} \mathrm{Cl}_{2}(5 \times 10 \mathrm{~mL})$ and dried under a flow of nitrogen to afford the resin-captured product. To the resin was added $\mathrm{CH}_{2} \mathrm{Cl}_{2}(3 \mathrm{~mL}) / \mathrm{MeOH}(1 \mathrm{~mL})$, and ozone was bubbled through the solution for $20 \mathrm{~min}$ at $0{ }^{\circ} \mathrm{C}$. Sodium borohydride $(100 \mathrm{mg})$ was added and the reaction was stirred at $\mathrm{rt}$ for $16 \mathrm{~h}$. The reaction mixture was transferred to an SPE cartridge, and was rinsed with $0.5 \mathrm{~N} \mathrm{HCl}(2 \times 2 \mathrm{~mL})$, THF $(2 \times 5 \mathrm{~mL})$, and $\mathrm{CH}_{2} \mathrm{Cl}_{2}(5 \times 5 \mathrm{~mL})$. The combined liquid phase was extracted with saturated aqueous $\mathrm{NaCl}(3 \times 10 \mathrm{~mL})$, and the organic layer was dried over $\mathrm{MgSO}_{4}$. Removal of the solvent under reduced pressure afforded 20 (16 mg, 39\% yield, $\mathrm{dr}>20: 1)$ as a pale yellow oil; ${ }^{1} \mathrm{H}$ NMR $\left(400 \mathrm{MHz}, \mathrm{CDCl}_{3}\right) \delta 7.48(\mathrm{~s}, 1 \mathrm{H}), 7.38(\mathrm{~d}, 1 \mathrm{H}, J=7.6 \mathrm{~Hz}), 7.27-7.13(\mathrm{~m}$, $2 \mathrm{H}), 4.61(\mathrm{~d},, 1 \mathrm{H} J=9 \mathrm{~Hz}), 4.56(\mathrm{~d}, 1 \mathrm{H}, J=9 \mathrm{~Hz}), 3.59-3.65(\mathrm{~m}, 3 \mathrm{H}), 3.44(\mathrm{~s}, 3 \mathrm{H}), 2.78(\mathrm{dd}, 1 \mathrm{H}$, $J=5.7 \mathrm{~Hz}, 2.4 \mathrm{~Hz}), 1.82-2.30(\mathrm{~m}, 3 \mathrm{H}), 1.6-1.8(\mathrm{~m}, 5 \mathrm{H}), 1.21-1.40(\mathrm{~m}, 5 \mathrm{H}), 1.02(\mathrm{~d}, 3 \mathrm{H}, J=5.4$ $\mathrm{Hz}), 0.91(\mathrm{~d}, 3 \mathrm{H}, J=5.4 \mathrm{~Hz}) ;{ }^{13} \mathrm{C}$ NMR $\left(100 \mathrm{MHz}, \mathrm{CDCl}_{3}\right) \delta 141.7,130.6,130.5,130.1,125.9$, 122.6, 85.9, 84.3, 74.0, 66.5, 61.2, 41.4, 38.1, 37.8, 30.3, 29.8, 26.7, 26.5, 26.4, 10.8, 10.4; IR (neat) $v_{\max }: 2924,2870,1558,1507,1457,1071,749 ;[\alpha]^{23}=-15.1^{\circ}\left(\mathrm{c}=0.20, \mathrm{CHCl}_{3}\right)$; CIHRMS $[\mathrm{M}+1]^{+}$calculated for $\mathrm{C}_{21} \mathrm{H}_{33} \mathrm{BrO}_{3}$ 413.1613, found 413.1710.

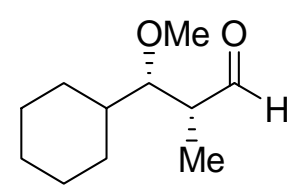

21

Aldehyde 21: Cyclohexanecarboxaldehyde (110 mg, $0.985 \mathrm{mmol})$, TMSOMe $(0.285 \mathrm{~mL}, 1.99 \mathrm{mmol})$ and anthracene-tagged organosilane $(S)-4(520 \mathrm{mg}, 1.11$ mmol) were dissolved in $\mathrm{CH}_{2} \mathrm{Cl}_{2}(6.0 \mathrm{~mL})$ and cooled to $-78{ }^{\circ} \mathrm{C}$. Triflic acid $(0.087 \mathrm{~mL}, 1.00 \mathrm{mmol})$ was added to the mixture at $-78{ }^{\circ} \mathrm{C}$, and the solution was warmed to $-60{ }^{\circ} \mathrm{C}$ and stirred at that temperature for $16 \mathrm{~h}$ and quenched with saturated aqueous $\mathrm{NaHCO}_{3}(4 \mathrm{~mL})$. The mixture was extracted with $\mathrm{CH}_{2} \mathrm{Cl}_{2}(3 \times 10 \mathrm{~mL})$ and the combined organic layers were dried over $\mathrm{MgSO}_{4}$. Removal of solvent under reduced pressure afforded a pale yellow oil. This material was added to a microwave reaction vessel, chlorobenzene $(4 \mathrm{~mL})$ and maleimide resin $7(200 \mathrm{mg}, 0.450 \mathrm{mmol}$, loading $1.54 \mathrm{mmol} / \mathrm{g})$ were added, and the mixture was irradiated for $20 \mathrm{~min}$ at $150{ }^{\circ} \mathrm{C}(200 \mathrm{~W})$. After cooling to $\mathrm{rt}$, the mixture was transferred to an SPE cartridge, and the resin was rinsed with MeOH $(3 \times 5 \mathrm{~mL})$, THF $(5 \times 5 \mathrm{~mL})$, $\mathrm{CH}_{2} \mathrm{Cl}_{2}(5 \times 10 \mathrm{~mL})$ and dried under a flow of nitrogen to afford the resin-captured product. To the resin was added in $\mathrm{CH}_{2} \mathrm{Cl}_{2}(6 \mathrm{~mL}) / \mathrm{MeOH}(0.5 \mathrm{~mL})$, and ozone was bubbled through the solution for $20 \mathrm{~min}$ at $0^{\circ} \mathrm{C}$. Excess $\mathrm{PMe}_{3}(15 \mathrm{~mL}, 1 \mathrm{M}$ solution in THF) was added and the reaction was allowed to stir at $\mathrm{rt}$ for $8 \mathrm{~h}$. The reaction mixture was concentrated under reduced pressure to yield the crude aldehyde product. The mixture was dissolved in $8 \mathrm{~mL}$ of $\mathrm{CH}_{2} \mathrm{Cl}_{2}$, transferred to an Isolute ${ }^{\circledR}$ cartridge pre-loaded with water, and eluted to remove the trimethylphosphine oxide via supported-liquid extraction. ${ }^{\mathrm{S} 5}$ Removal of $\mathrm{CH}_{2} \mathrm{Cl}_{2}$ under reduced pressure yielded aldehyde $\mathbf{2 1}$ (114mg, 61\% yield, $\mathrm{dr}>20: 1) ;{ }^{1} \mathrm{H}$ NMR $\left(400 \mathrm{MHz}, \mathrm{CDCl}_{3}\right) \delta 9.80(\mathrm{~d}, 1 \mathrm{H}, J=1.2 \mathrm{~Hz}), 3.37-3.40$ $(\mathrm{m}, 1 \mathrm{H}), 3.35(\mathrm{~s}, 3 \mathrm{H}), 2.50-2.54(\mathrm{~m}, 1 \mathrm{H}), 1.96-1.93(\mathrm{~m}, 1 \mathrm{H}), 1.78-1.75(\mathrm{~m}, 2 \mathrm{H}), 1.69-1.51(\mathrm{~m}, 2 \mathrm{H})$,

\footnotetext{
S5 (a) Johnson, C. R.; Zhang, B.; Fantauzzi, P.; Hocker, M.; Yager, K. M. Tetrahedron 1998, 54, 4097. (b) Breitenbucher, J. G.; Arienti, K. L.; McClure, K. J. J. Comb. Chem. 2001, 3, 528.
} 
1.51-1.48 (m, 2H), 1.26-1.16 (m, 2H), $1.13(\mathrm{~d}, 3 \mathrm{H}, J=6.8 \mathrm{~Hz}), 1.10-1.01(\mathrm{~m}, 2 \mathrm{H}) ;{ }^{13} \mathrm{C}$ NMR $(100$ $\left.\mathrm{MHz}, \mathrm{CDCl}_{3}\right) \delta 205.1,82.7,60.1,44.7,40.3,30.2,29.4,26.6,26.5,26.4,9.1$; IR (neat) $v_{\max }: 2927$, $2852,1727,1448,1193,1109,973,944,890 ;[\alpha]^{23}{ }_{\mathrm{D}}=-29.2^{\circ}\left(\mathrm{c}=0.80, \mathrm{CHCl}_{3}\right)$.

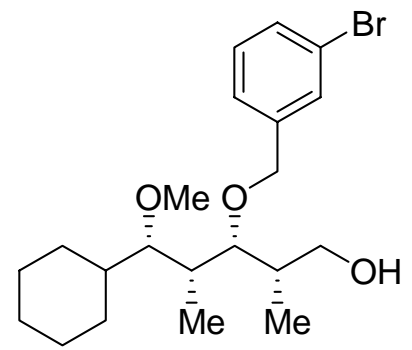

22

Alcohol 22: Aldehyde 21 (20 mg, $0.108 \mathrm{mmol})$ and silane (S)-4 (94 mg, $0.228 \mathrm{mmol})$ were dissolved in $\mathrm{CH}_{2} \mathrm{Cl}_{2}(1 \mathrm{~mL})$ and cooled to $-60{ }^{\circ} \mathrm{C}$. Triflic acid $(13 \mu \mathrm{L}, 0.100 \mathrm{mmol})$ was added to the mixture at $-60{ }^{\circ} \mathrm{C}$, and the solution was stirred at $-60{ }^{\circ} \mathrm{C}$ for $48 \mathrm{~h}$ before saturated $\mathrm{NaHCO}_{3}(2$ $\mathrm{mL})$ was added. The mixture was extracted with $\mathrm{CH}_{2} \mathrm{Cl}_{2}(3 \times 10 \mathrm{~mL})$ and the combined organic layers were dried over $\mathrm{MgSO}_{4}$. Removal of the solvent under reduced pressure afforded a pale yellow oil. This material was charged to a microwave reaction vessel, chlorobenzene $(4 \mathrm{~mL})$ and maleimide resin 7 (400 $\mathrm{mg}, 0.450 \mathrm{mmol}$, loading $1.54 \mathrm{mmol} / \mathrm{g}$ ) were added, and the mixture was irradiated for $20 \mathrm{~min}$ at $150{ }^{\circ} \mathrm{C}(200 \mathrm{~W})$. The cooled reaction mixture was transferred to an SPE cartridge, and the resin rinsed with $\mathrm{MeOH}(3 \times 5 \mathrm{~mL})$, THF $(5 \times 5 \mathrm{~mL})$, and $\mathrm{CH}_{2} \mathrm{Cl}_{2}(5 \times 10 \mathrm{~mL})$ and dried under a flow of nitrogen to yield the resin-captured product. To the resin was added $\mathrm{CH}_{2} \mathrm{Cl}_{2}(3 \mathrm{~mL}) / \mathrm{MeOH}(1 \mathrm{~mL})$ and ozone was bubbled through the solution for $20 \mathrm{~min}$ at $0{ }^{\circ} \mathrm{C}$. Sodium borohydride $(100 \mathrm{mg})$ was added and the reaction was allowed to stir at $\mathrm{rt}$ for $16 \mathrm{~h}$. The reaction mixture was transferred to an SPE cartridge, and was rinsed with $0.5 \mathrm{~N} \mathrm{HCl}(2 \times 2 \mathrm{~mL})$, THF $(2 \times 5 \mathrm{~mL}) \mathrm{CH}_{2} \mathrm{Cl}_{2}(5 \times 5 \mathrm{~mL})$. The combined liquid phase was washed with saturated aqueous $\mathrm{NaCl}(3 \times 10 \mathrm{~mL})$, and the organic layer dried over $\mathrm{MgSO}_{4}$. Removal of solvent under reduced pressure afforded 22 as an oil $(21 \mathrm{mg}, 47 \%$ yield, $\mathrm{dr}>20: 1) ;{ }^{1} \mathrm{H}$ NMR (400 $\mathrm{MHz}, \mathrm{CDCl}_{3}$ ) $\delta 7.48(\mathrm{~s}, 1 \mathrm{H}), 7.38(\mathrm{~d}, 1 \mathrm{H}, J=7.6 \mathrm{~Hz}), 7.27-7.13(\mathrm{~m}, 2 \mathrm{H}), 4.61(\mathrm{~d}, 1 \mathrm{H}, J=9 \mathrm{~Hz}), 4.56(\mathrm{~d}, 1 \mathrm{H}, J$ $=9 \mathrm{~Hz}), 3.59-3.65(\mathrm{~m}, 3 \mathrm{H}), 3.44(\mathrm{~s}, 3 \mathrm{H}), 2.78(\mathrm{dd}, 1 \mathrm{H}, J=5.7 \mathrm{~Hz}, 2.4 \mathrm{~Hz}), 1.82-2.30(\mathrm{~m}, 3 \mathrm{H})$, 1.6-1.8 (m, 5H), 1.21-1.40 (m, 5H), $1.02(\mathrm{~d}, 3 \mathrm{H}, J=5.4 \mathrm{~Hz}), 0.91(\mathrm{~d}, 3 \mathrm{H}, J=5.4 \mathrm{~Hz}) .{ }^{13} \mathrm{C}$ NMR $\left(100 \mathrm{MHz}, \mathrm{CDCl}_{3}\right) \delta 141.5,130.7,130.6,130.2,126.1,122.1,87.3,81.8,73.9,66.3,61.5,41.3$, $38.3,38.1,30.1,29.8,26.7,26.5,26.4,11.0,10.3$; IR (neat) $v_{\max }: 2924,2873,1556,1507,1457$, 1071,$749 ;[\alpha]^{23}=-11.7^{\circ}\left(\mathrm{c}=0.15, \mathrm{CHCl}_{3}\right)$.

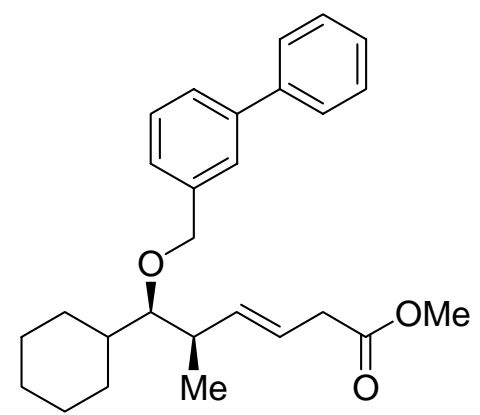

25a
Ester 25a: Cyclohexanecarboxaldehyde (23 mg, $0.214 \mathrm{mmol}$ ), 3-bromobenzyloxytrimethylsilane (104 $\mathrm{mg}, 0.398 \mathrm{mmol})$ and $(R)-4$ (103 mg, $0.22 \mathrm{mmol}$ ) were dissolved in $\mathrm{CH}_{2} \mathrm{Cl}_{2}(6.0 \mathrm{~mL})$ and cooled to $-78{ }^{\circ} \mathrm{C}$. Triflic acid $(0.017 \mathrm{~mL}, 0.20 \mathrm{mmol})$ was added to the mixture at $-78{ }^{\circ} \mathrm{C}$, and the solution was warmed to $-60{ }^{\circ} \mathrm{C}$ and stirred at that temperature for $24 \mathrm{~h}$ before being quenched by addition of saturated $\mathrm{NaHCO}_{3}(1 \mathrm{~mL})$. The mixture was extracted with $\mathrm{CH}_{2} \mathrm{Cl}_{2}$ $(3 \times 5 \mathrm{~mL})$ and the combined organic layers were dried over $\mathrm{MgSO}_{4}$ and the solvent removed under reduced pressure to afford a pale

yellow oil. 
This material was added to a microwave reaction vessel, chlorobenzene $(4 \mathrm{~mL})$ and maleimide resin 7 (200 $\mathrm{mg}, 0.180 \mathrm{mmol}$, loading $1.54 \mathrm{mmol} / \mathrm{g}$ ) were added, and the mixture was irradiated for $20 \mathrm{~min}$ at $150{ }^{\circ} \mathrm{C}(200 \mathrm{~W})$. After cooling to $\mathrm{rt}$, the reaction mixture was transferred to an SPE cartridge, and the solid phase was rinsed with $\mathrm{MeOH}(3 \times 5 \mathrm{~mL})$, THF ( $5 \times 5 \mathrm{~mL}), \mathrm{CH}_{2} \mathrm{Cl}_{2}$ $(5 \times 10 \mathrm{~mL})$ and dried under a flow of nitrogen to yield the resin-captured product.

Tetrakis(triphenylphosphine)palladium (0) $(22.6 \mathrm{mg}, 0.02 \mathrm{mmol})$ was weighed into a 10 $\mathrm{mL}$ microwave reaction tube followed by addition of NMP $(1 \mathrm{~mL})$ and the aforementioned resin $(200 \mathrm{mg})$. After addition of phenyltributyltin $(300 \mathrm{mg}, 2.00 \mathrm{mmol})$, the microwave tube was sealed and heated at $80{ }^{\circ} \mathrm{C}$ for $16 \mathrm{~h}$. The cooled reaction mixture was transferred to an SPE cartridge, and the resin was rinsed with $\mathrm{MeOH}(3 \times 2 \mathrm{~mL})$, THF $(5 \times 2 \mathrm{~mL})$, and $\mathrm{CH}_{2} \mathrm{Cl}_{2}(5 \times 3 \mathrm{~mL})$ and dried under a flow of nitrogen. To the resin was added THF $(2 \mathrm{~mL}) / \mathrm{MeOH}(0.5 \mathrm{~mL})$, freshly prepared 1 $\mathrm{M} \mathrm{NaOMe}(0.4 \mathrm{~mL}, 0.4 \mathrm{mmol})$ was added, and the mixture was agitated for $2 \mathrm{~h}$. The reaction mixture was transferred to an SPE cartridge and was rinsed with $0.5 \mathrm{~N} \mathrm{HCl}(2 \times 1 \mathrm{~mL}), \mathrm{THF}(2 \times 2$ $\mathrm{mL})$, and $\mathrm{CH}_{2} \mathrm{Cl}_{2}(5 \times 2 \mathrm{~mL})$. The combined organic layer was extracted with saturated $\mathrm{NaCl}$ solution $(3 \times 3 \mathrm{~mL})$, and the organic layer dried over $\mathrm{MgSO}_{4}$. Removal of solvent under reduced pressure afforded ester 25a (38 mg, 46\% yield); ${ }^{1} \mathrm{H}$ NMR $\left(400 \mathrm{MHz}, \mathrm{CDCl}_{3}\right) \delta 7.56-7.60(\mathrm{~m}, 3 \mathrm{H})$, 7.32-7.52 (m, 6H), 5.56-5.59 (m, 2H), $4.61(\mathrm{~s}, 2 \mathrm{H}), 3.65(\mathrm{~s}, 3 \mathrm{H}), 3.01-3.04(\mathrm{~m}, 3 \mathrm{H}), 2.49-2.51(\mathrm{~m}$, $1 \mathrm{H}), 1.89-1.90(\mathrm{~m}, 1 \mathrm{H}), 1.60-1.79(\mathrm{~m}, 5 \mathrm{H}), 1.12-1.21(\mathrm{~m}, 5 \mathrm{H}), 1.08(\mathrm{~d}, 3 \mathrm{H}, J=6.4 \mathrm{~Hz}) .{ }^{13} \mathrm{C}$ NMR $\left(100 \mathrm{MHz}, \mathrm{CDCl}_{3}\right) \delta 172.6,144.7,141.5,141.4,139.8,139.1,138.8,128.9,127.4,126.7,126.6$, $126.4,121.0,88.5,75.4,51.9,41.2,39.3,38.2,30.8,28.5,26.8,26.7,26.5,15.4$; IR (neat) $v_{\max }$ : $2925,2851,1737,1456,1075,913,749 ;[\alpha]_{\mathrm{D}}^{23}=9.4\left(\mathrm{c}=0.10, \mathrm{CHCl}_{3}\right)$; CIHRMS $[\mathrm{M}]^{+}$ calculated for $\mathrm{C}_{27} \mathrm{H}_{34} \mathrm{O}_{3} 406.2508$, found 406.2539 .

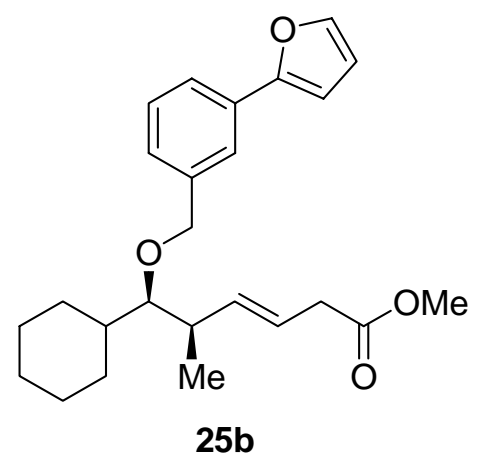

$+17.3\left(\mathrm{c}=0.10, \mathrm{CHCl}_{3}\right)$.

Ester 25b: Prepared using 2-(tributylstannyl)-furan (45\% yield).

${ }^{1} \mathrm{H}$ NMR $\left(400 \mathrm{MHz}, \mathrm{CDCl}_{3}\right) \delta 7.63(\mathrm{~s}, 1 \mathrm{H}), 7.59(\mathrm{~d}, 1 \mathrm{H}, J=8 \mathrm{~Hz})$, $7.49(\mathrm{~s}, 1 \mathrm{H}), 7.27-7.39(\mathrm{~m}, 2 \mathrm{H}), 6.63(\mathrm{~d}, 1 \mathrm{H}, J=2 \mathrm{~Hz}), 6.49-6.50(\mathrm{~m}$, $1 \mathrm{H}), 5.57-5.59(\mathrm{~m}, 2 \mathrm{H}), 4.57(\mathrm{~s}, 2 \mathrm{H}), 3.65(\mathrm{~s}, 3 \mathrm{H}), 3.01-3.04(\mathrm{~m}, 3 \mathrm{H})$, 2.49-2.51 (m, 1H), 1.89-1.90 (m,1H), 1.60-1.79 (m, 5H), 1.12- 1.21 $(\mathrm{m}, 5 \mathrm{H}), 1.09(\mathrm{~d}, 3 \mathrm{H}, J=6.4 \mathrm{~Hz}) ;{ }^{13} \mathrm{C} \mathrm{NMR}\left(100 \mathrm{MHz}, \mathrm{CDCl}_{3}\right) \delta$ $172.8,142.2$, 138.8, 128.8, 126.7, 123.1, 123.0, 121.0, 111.8, 105.2, $88.5,75.2,51.9,41.2,39.3,38.2,30.7,28.5,26.8,26.7,26.5,15.3$; IR (neat) $v_{\max }: 3042,2928,2851,1456,1075,912,746 ;[\alpha]^{23}{ }_{\mathrm{D}}=$<smiles>CO[C@H](c1ccccc1)[C@@H](C)[C@H](OC)[C@@H](C)COC(=O)C/C=C/[C@@H](C)[C@H](OCc1cccc(Br)c1)C1CCCCC1</smiles>

Ester 28: To a solution of 12 (12 $\mathrm{mg}, 0.047 \mathrm{mmol})$ and 26 (20 mg, $0.048 \mathrm{mmol})$ in $0.5 \mathrm{~mL}$ of chlorobenzene was added fluorous tin oxide 27 (4 
$\mathrm{mg}, 0.005 \mathrm{mmol}) .{ }^{\mathrm{S} 6}$ The resulting mixture was irradiated in a microwave reaction vessel for 20 minutes at $160{ }^{\circ} \mathrm{C}(150 \mathrm{~W})$. After cooling to $\mathrm{rt}$, the reaction mixture was concentrated in vacuo and the crude material was dissolved in $250 \mu \mathrm{L}$ of $\mathrm{MeOH}$. The solution was applied to a fluorous silica catridge and was eluted with $5 \mathrm{~mL} \mathrm{MeOH} /$ water (90:10) using a AutoChem MiniBlock SPE rack. The eluent was collected and evaporated under vacuum to afford ester $28(27 \mathrm{mg}, 92 \%$ yield $) ;{ }^{1} \mathrm{H}$ NMR (400 MHz, $\left.\mathrm{CDCl}_{3}\right) \delta$ 7.29-7.49 (m, 4H), 7.17-7.26 (m, 5H), 5.52-5.58 (m, 2H), $4.51(\mathrm{~s}, 2 \mathrm{H})$, $4.01(\mathrm{~d}, 1 \mathrm{H}, J=6 \mathrm{~Hz}), 3.99(\mathrm{~d}, 2 \mathrm{H}, J=3.4 \mathrm{~Hz}), 3.35(\mathrm{~s}, 3 \mathrm{H}), 3.32-3.34(\mathrm{~m}, 1 \mathrm{H}), 3.19(\mathrm{~s}, 3 \mathrm{H})$, 2.98-3.02 ( m, 4H), 2.43-2.49 (m, 1H), 2.13-2.20 (m, 1H), 1.87-1.93 (m, 1H), 1.12- 1.21 (m, 5H), $0.93(\mathrm{~d}, J=6 \mathrm{~Hz}, 3 \mathrm{H}), 0.89(\mathrm{~d}, J=5.4 \mathrm{~Hz}, 3 \mathrm{H}), 0.85(\mathrm{~d}, J=6 \mathrm{~Hz}, 3 \mathrm{H}) ;{ }^{13} \mathrm{C}$ NMR $(100 \mathrm{MHz}$, $\left.\mathrm{CDCl}_{3}\right) \delta 172.2,142.3,141.0,138.6,130.6,130.1,128.5,127.6,127.4,125.8,126.1,122.6,121.3$, $88.7,85.0,82.5,74.5,67.4,60.9,57.4,43.2,41.2,39.3,38.4,35.5,32.2,30.7,30.4,29.6,28.5$, 26.8, 26.7, 26.4, 14.3, 11.6, 10.3; IR (neat) $v_{\max }$ : 2986, 2924, 2853, 1737, 1158, 1090, 751, 702, $746 ;[\alpha]^{23}=-14.7\left(\mathrm{c}=0.20, \mathrm{CHCl}_{3}\right)$; CIHRMS $[\mathrm{M}]^{+}$calculated for $\mathrm{C}_{35} \mathrm{H}_{49} \mathrm{BrO}_{5} 628.2763$, found 628.2778 .

${ }^{\text {S6 }}$ Bucher, B.; Curran, D. P. Tetrahedron Lett. 2000, 41, 9617. 


\section{Representative NMR spectra}
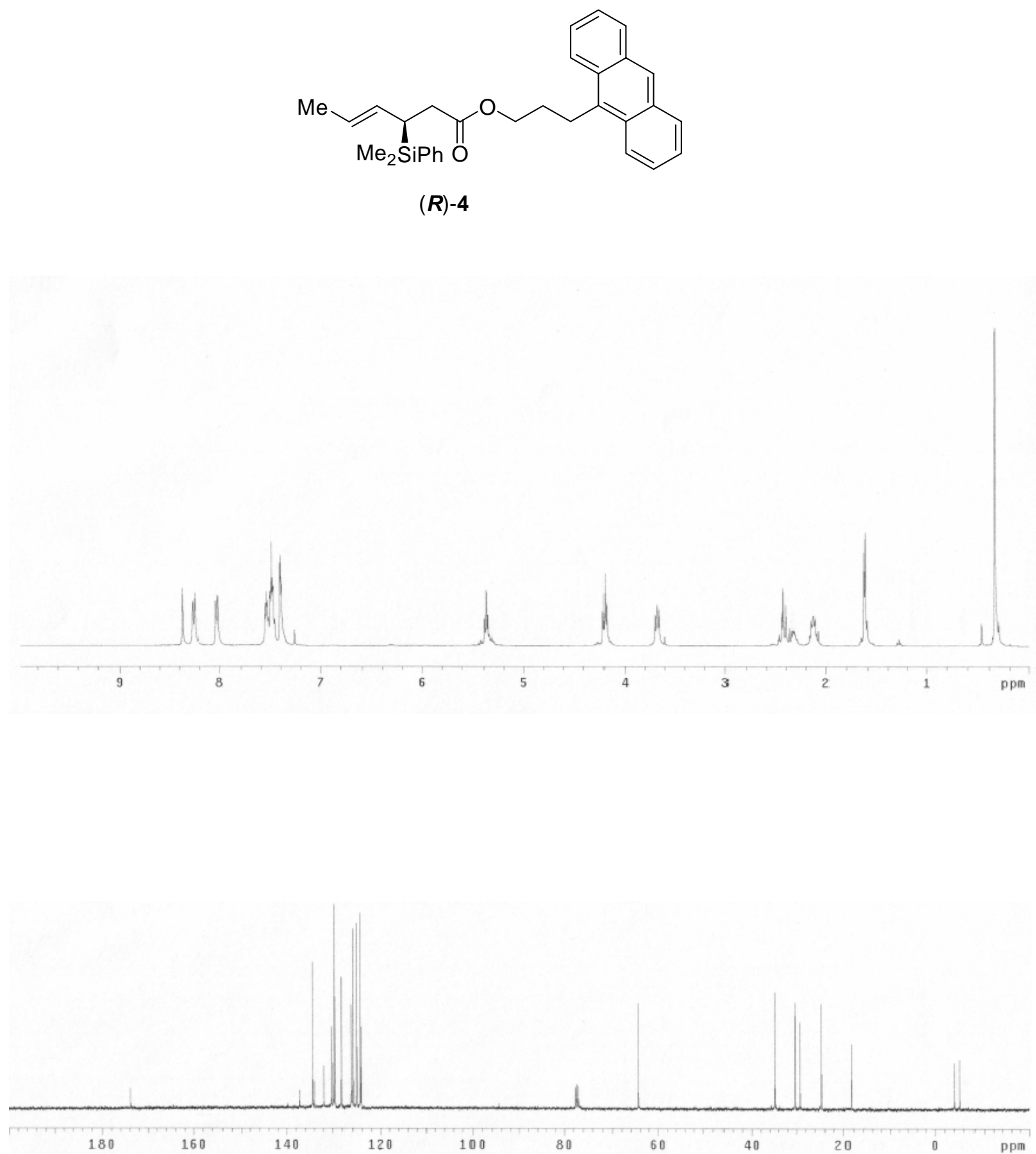

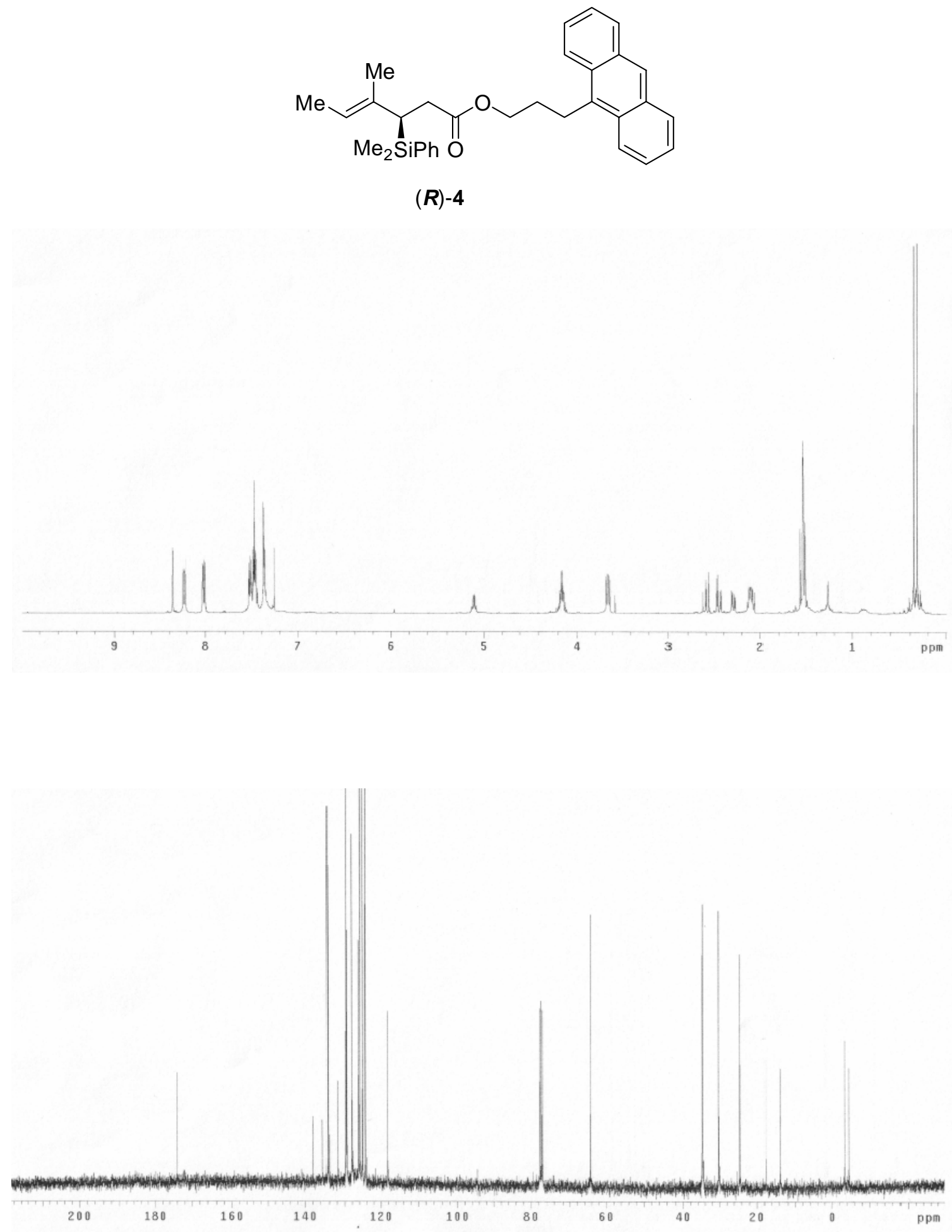


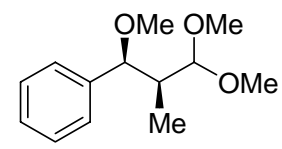

9
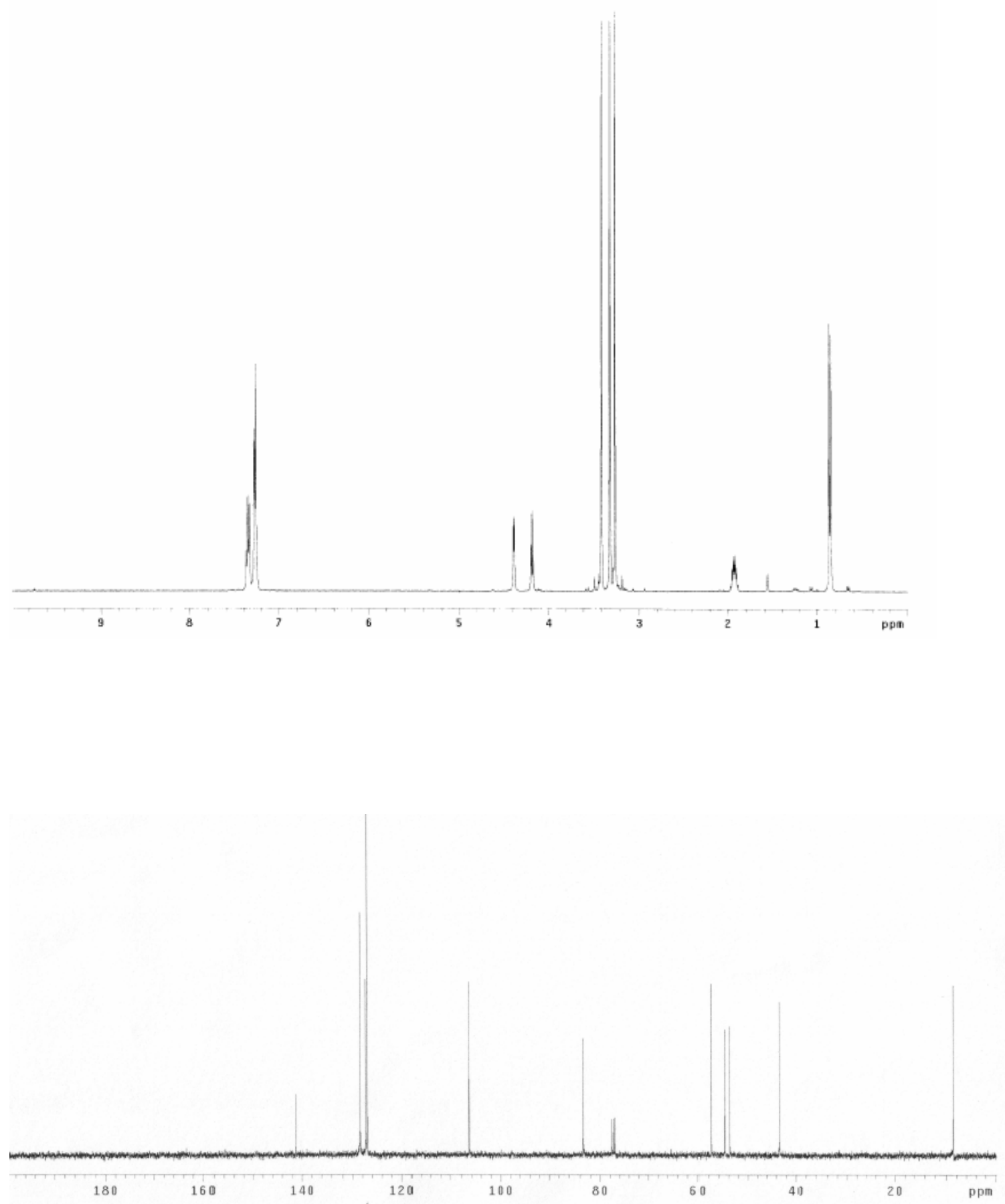

SI-17 

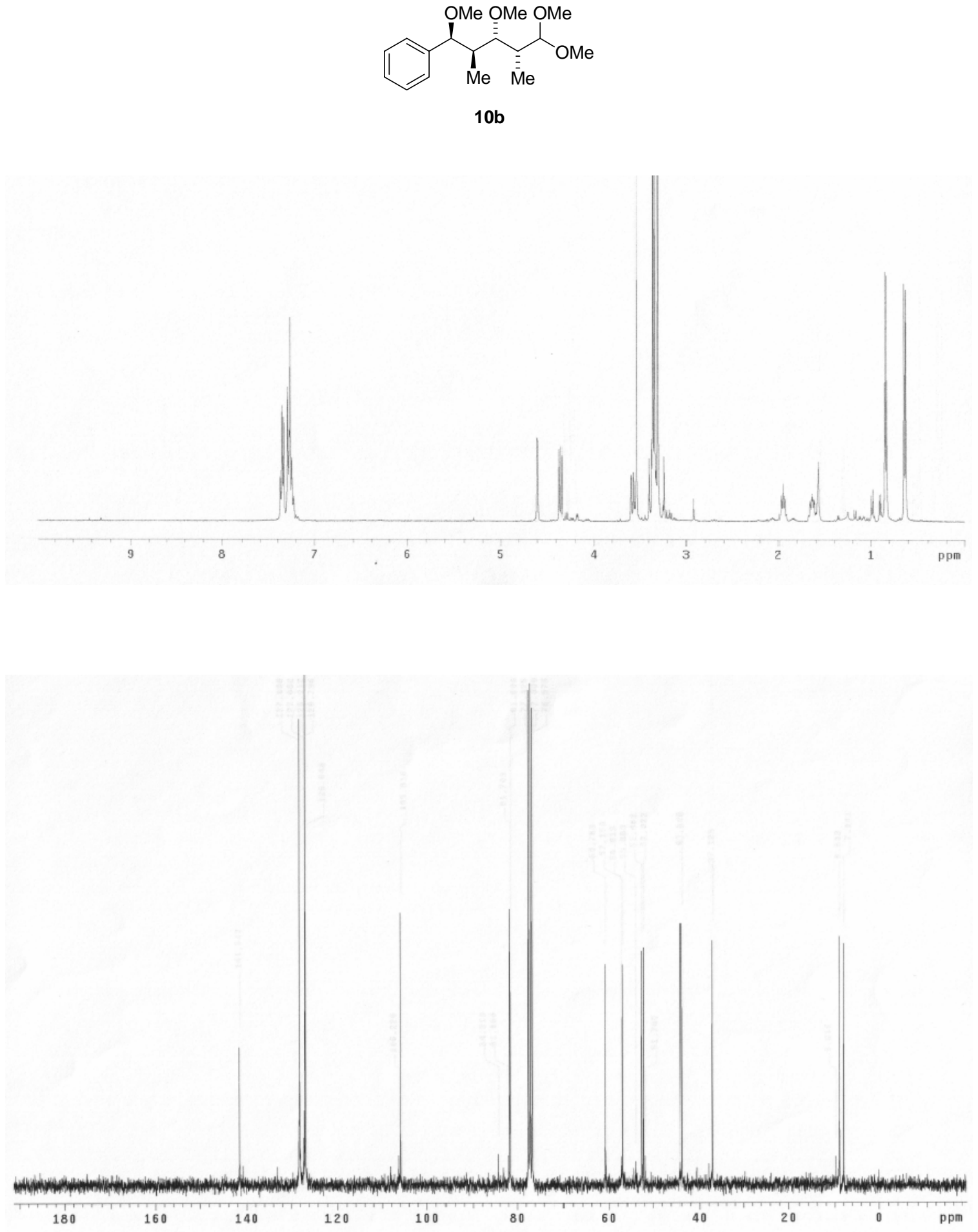

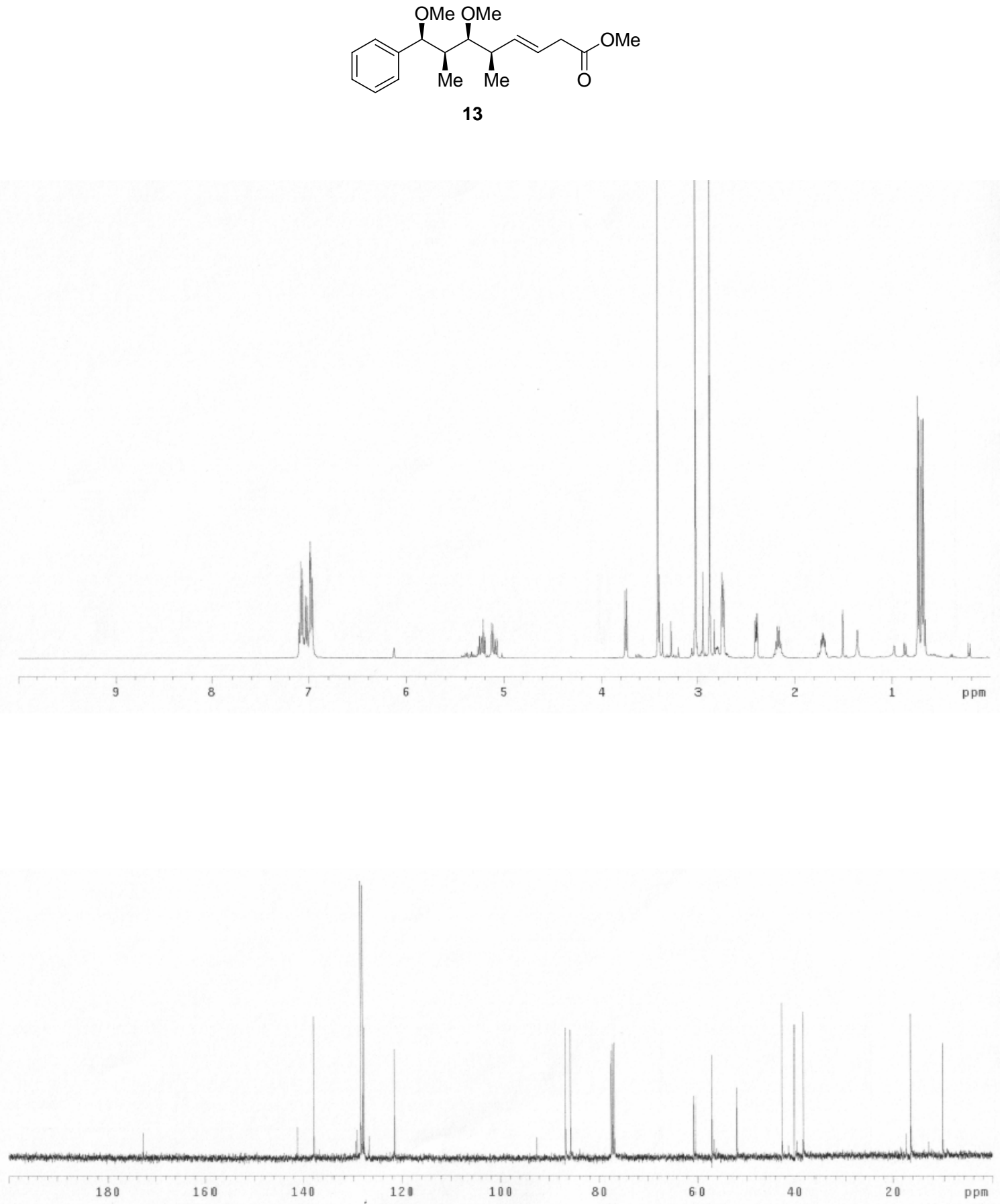

SI-19 


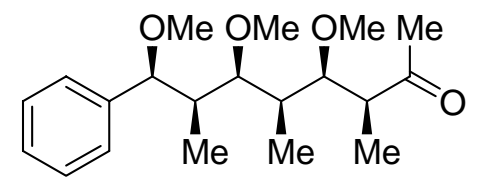

$15 a$
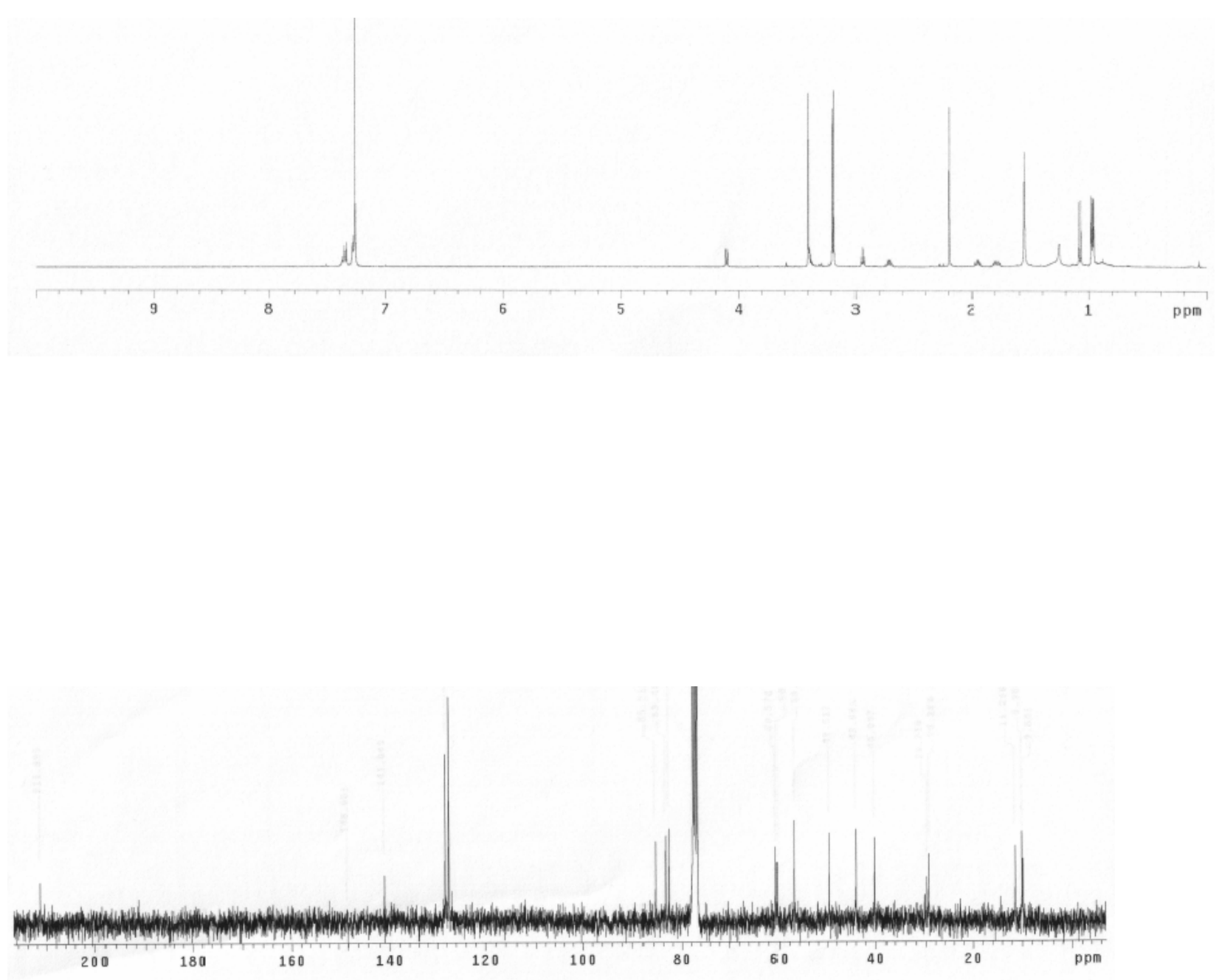

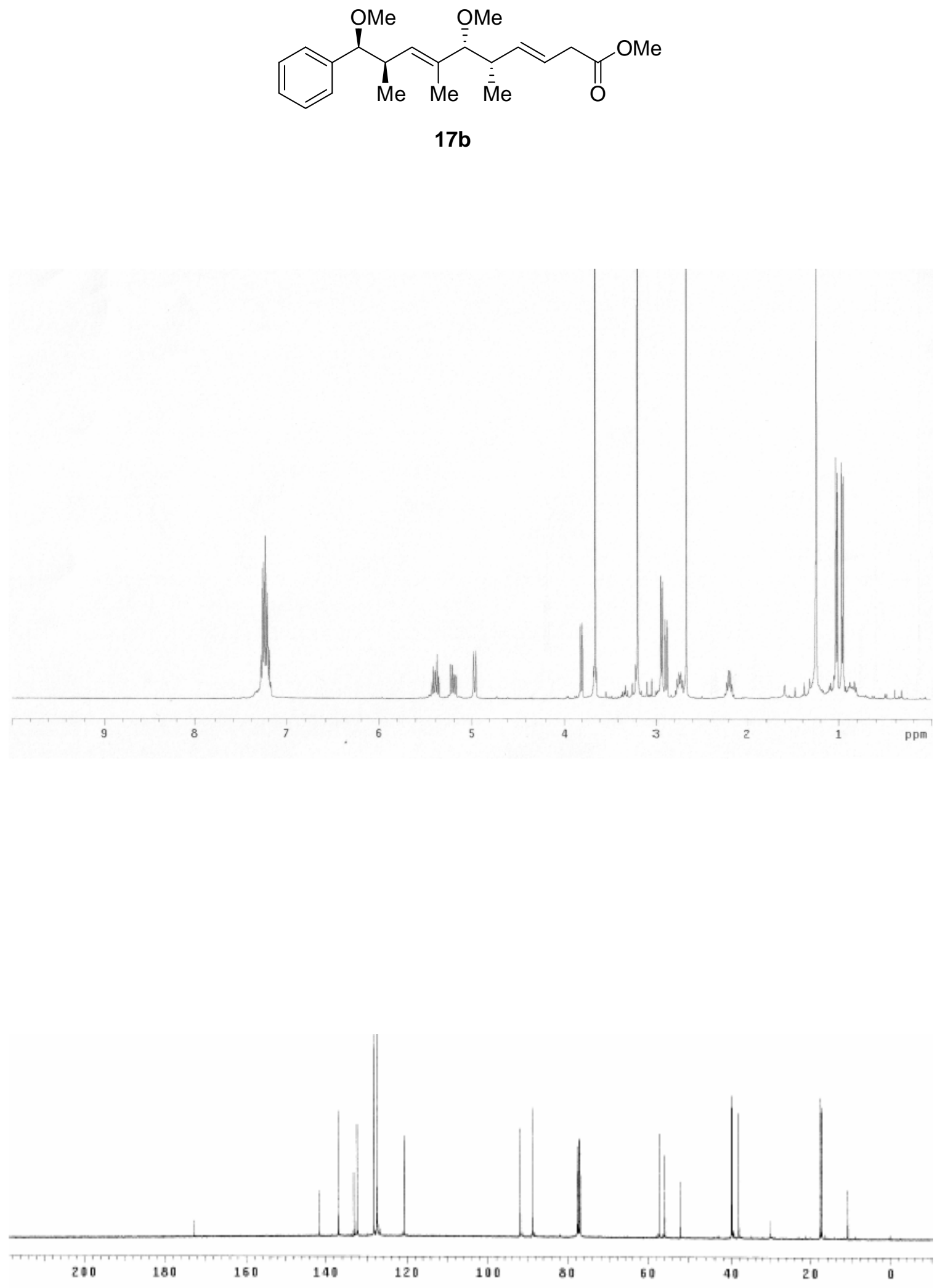


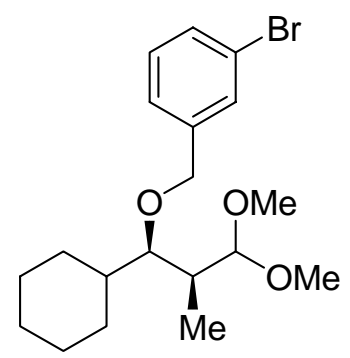

19
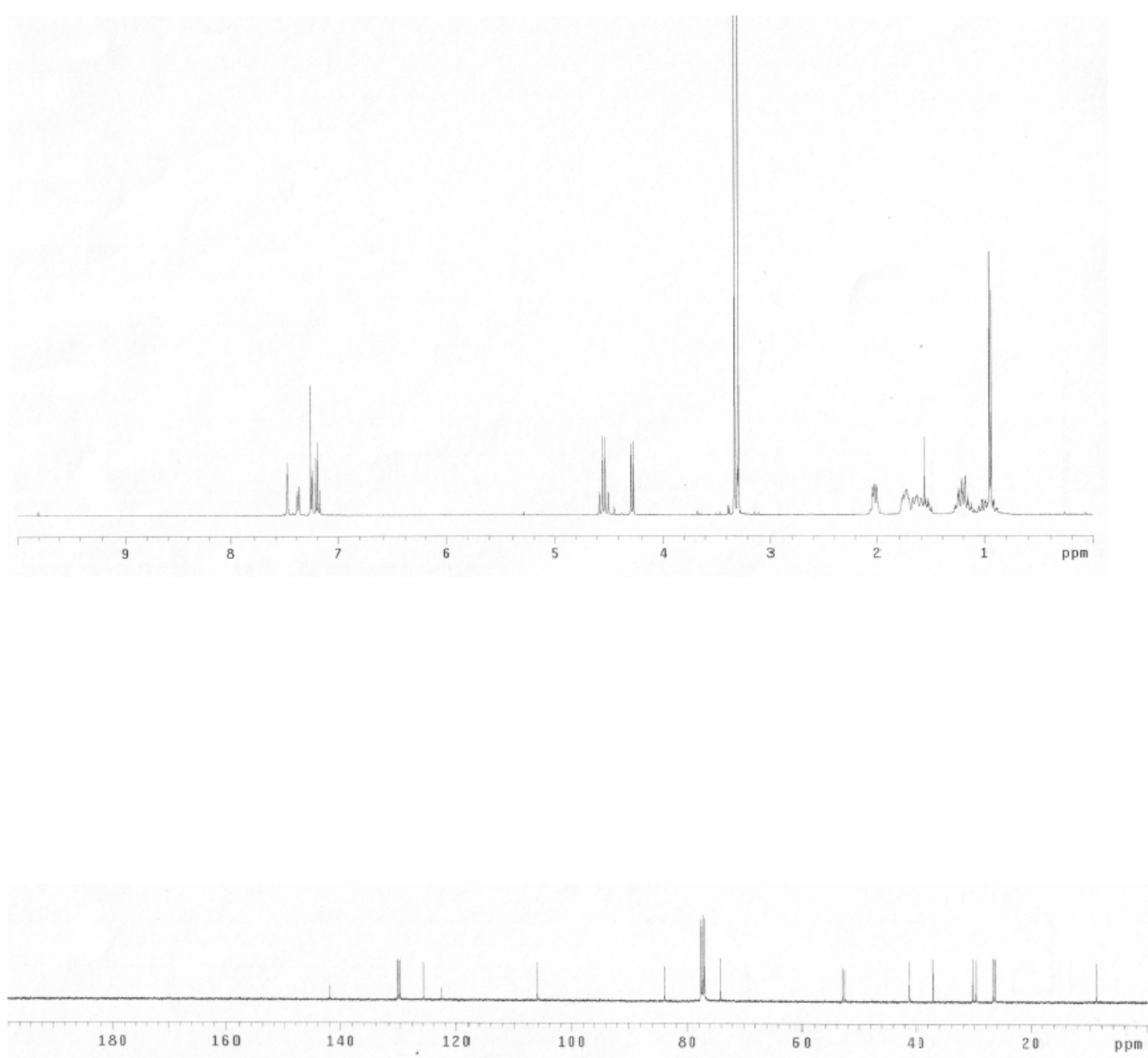


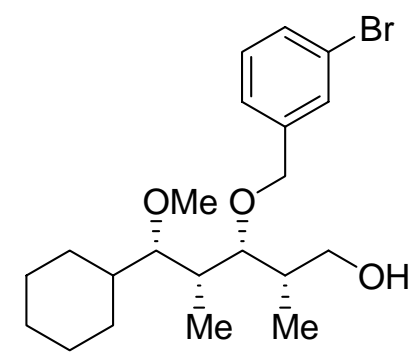

22
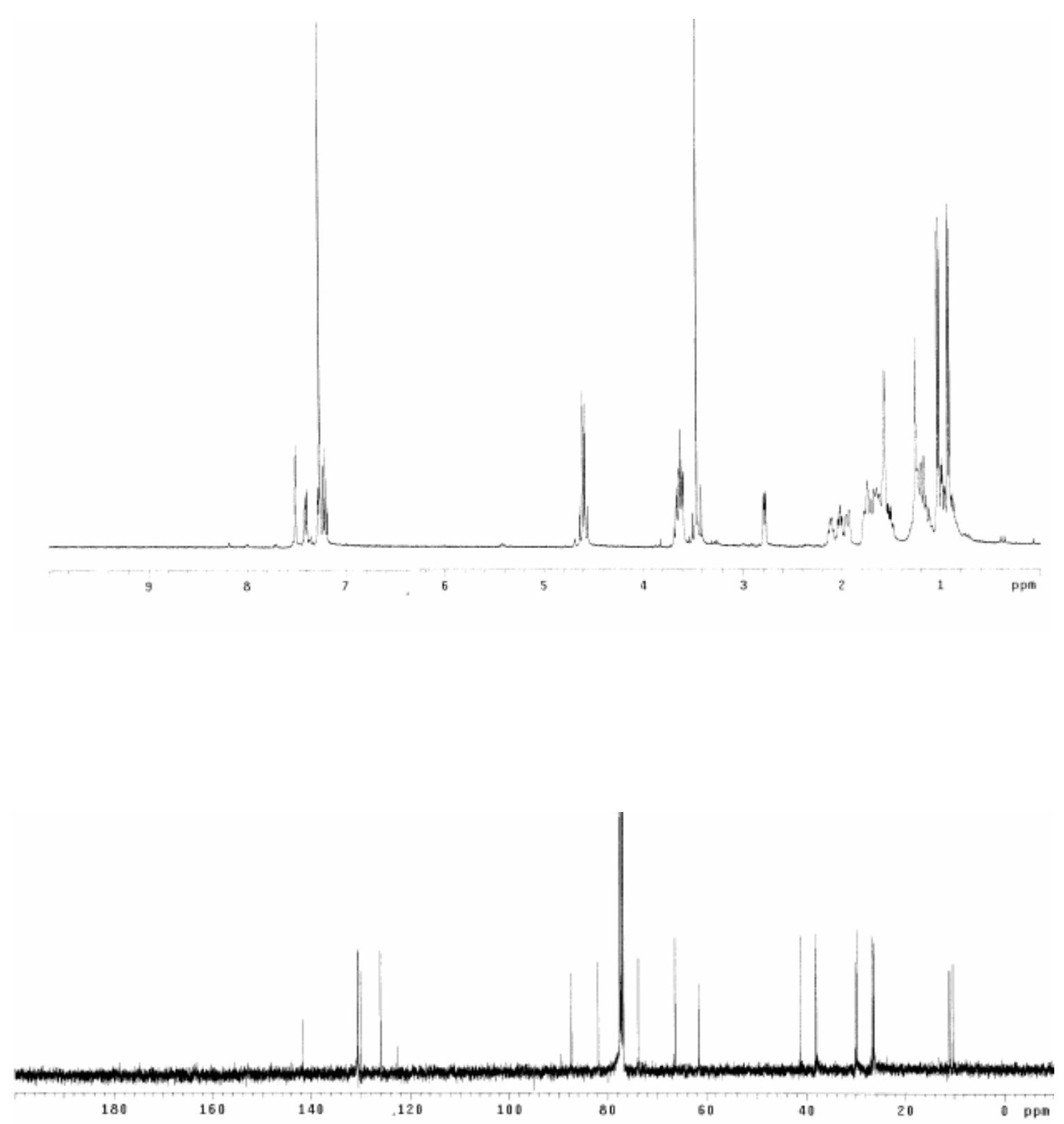


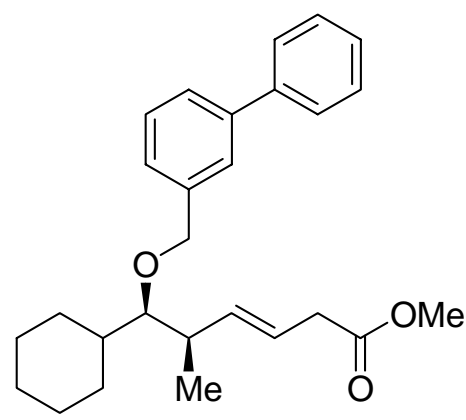

$25 a$
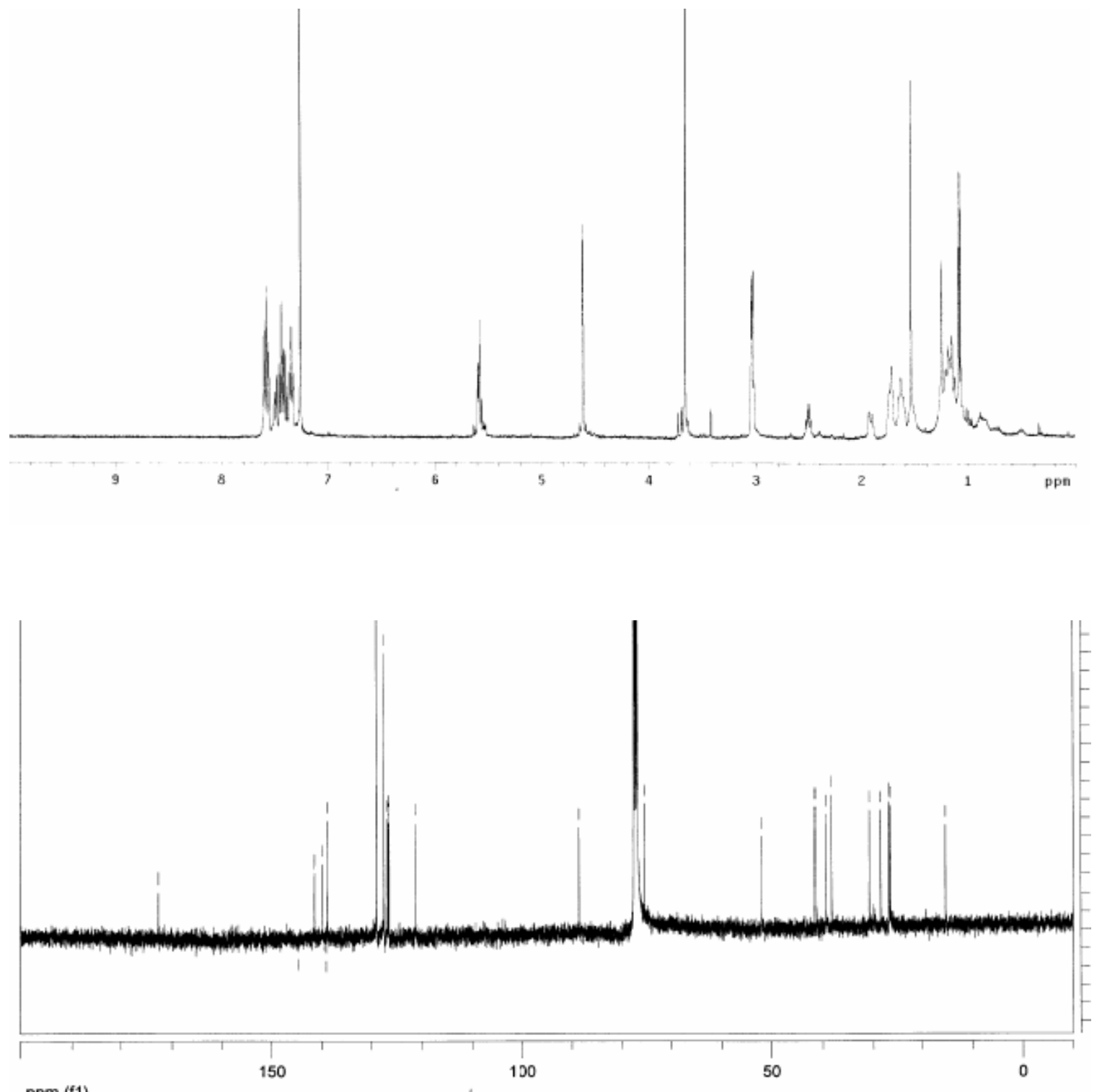

ppm (f1) 


\section{ELSD Chromatograms for Representative Compounds}

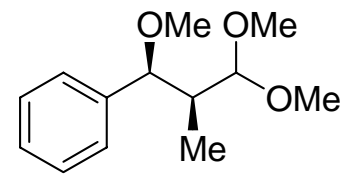

9

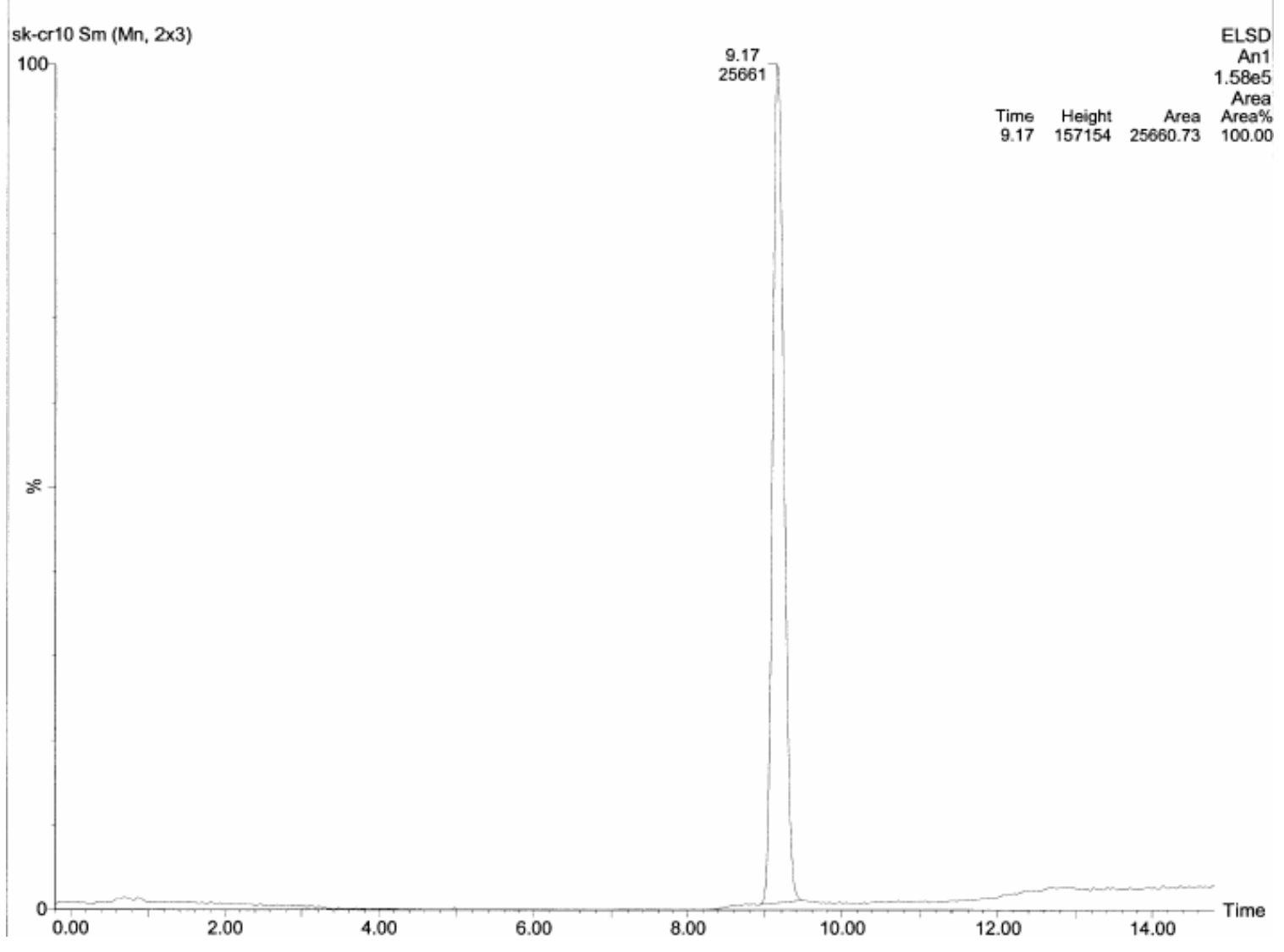




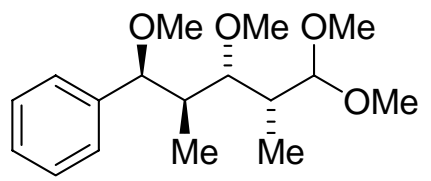

10b

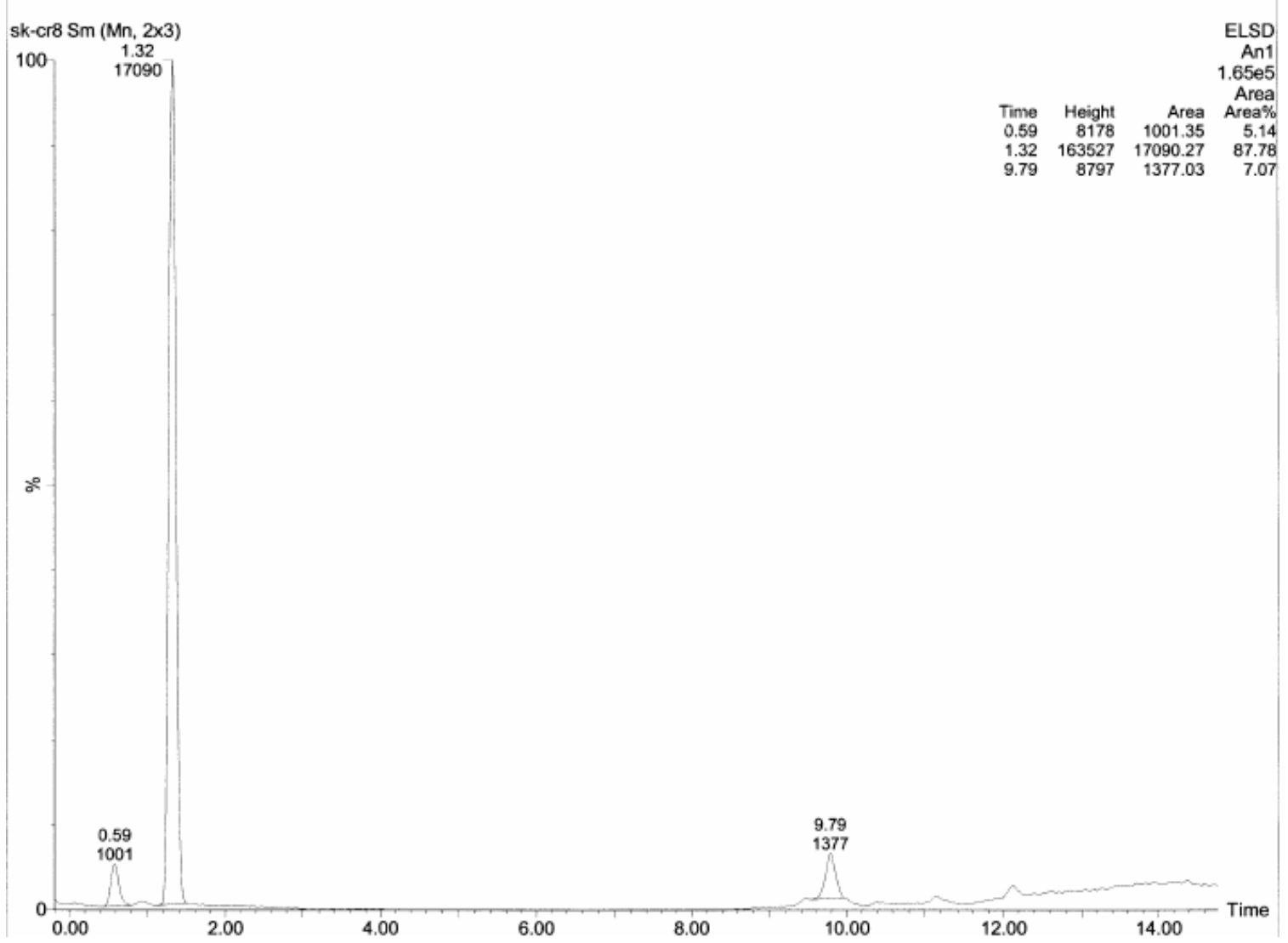



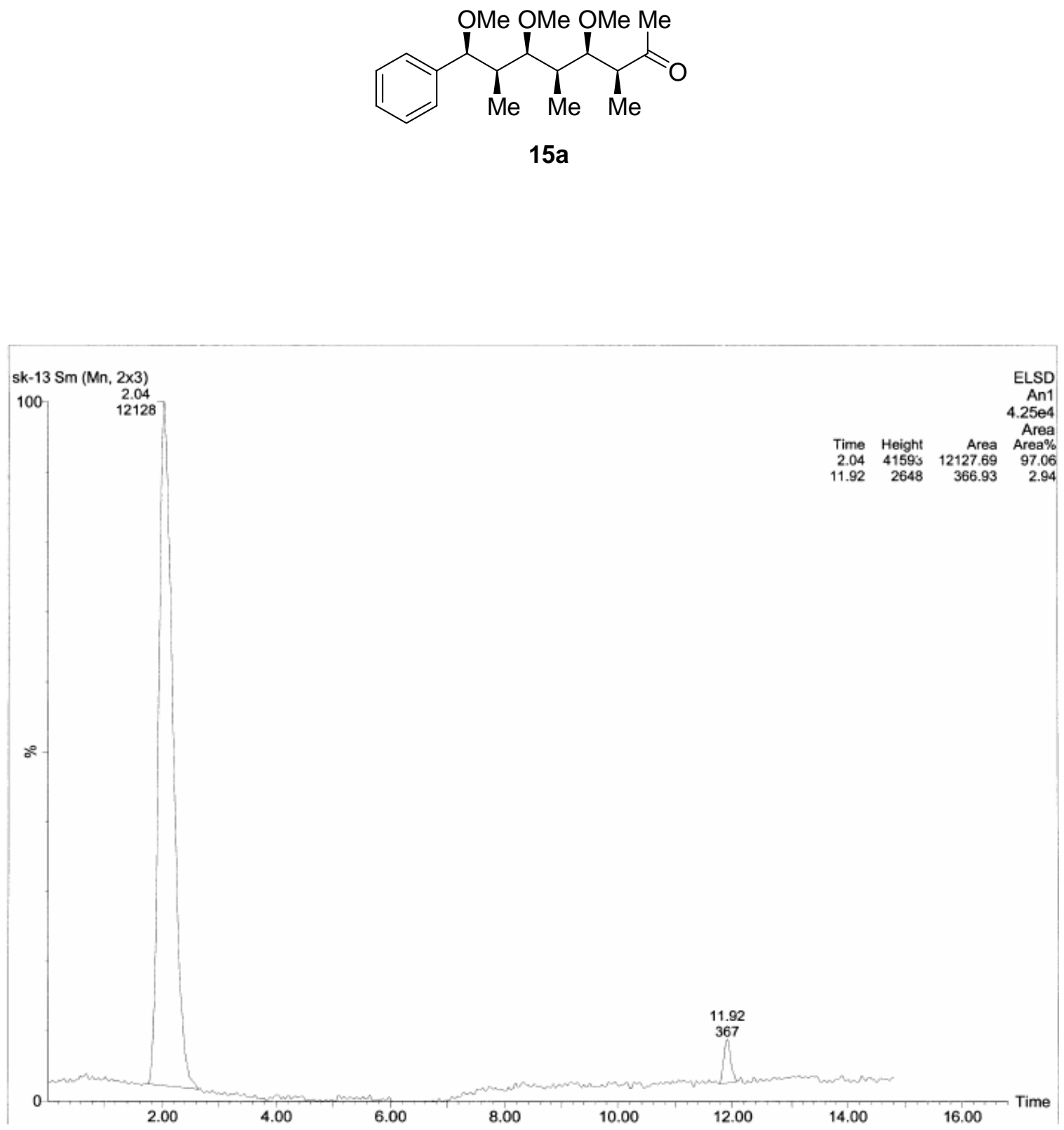


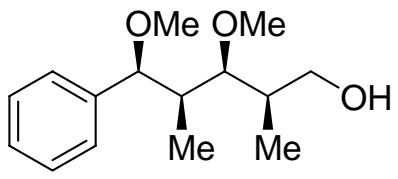

12

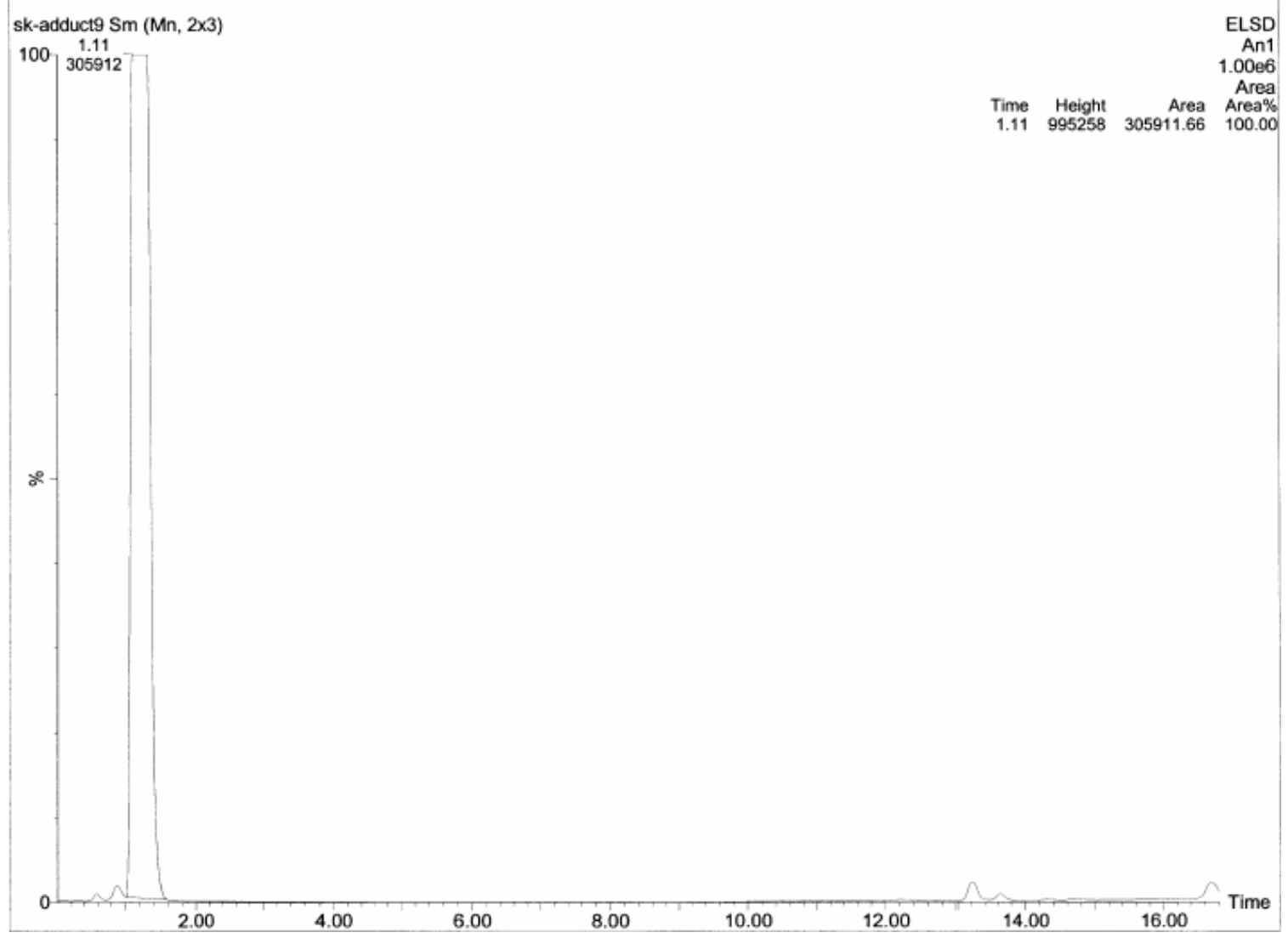



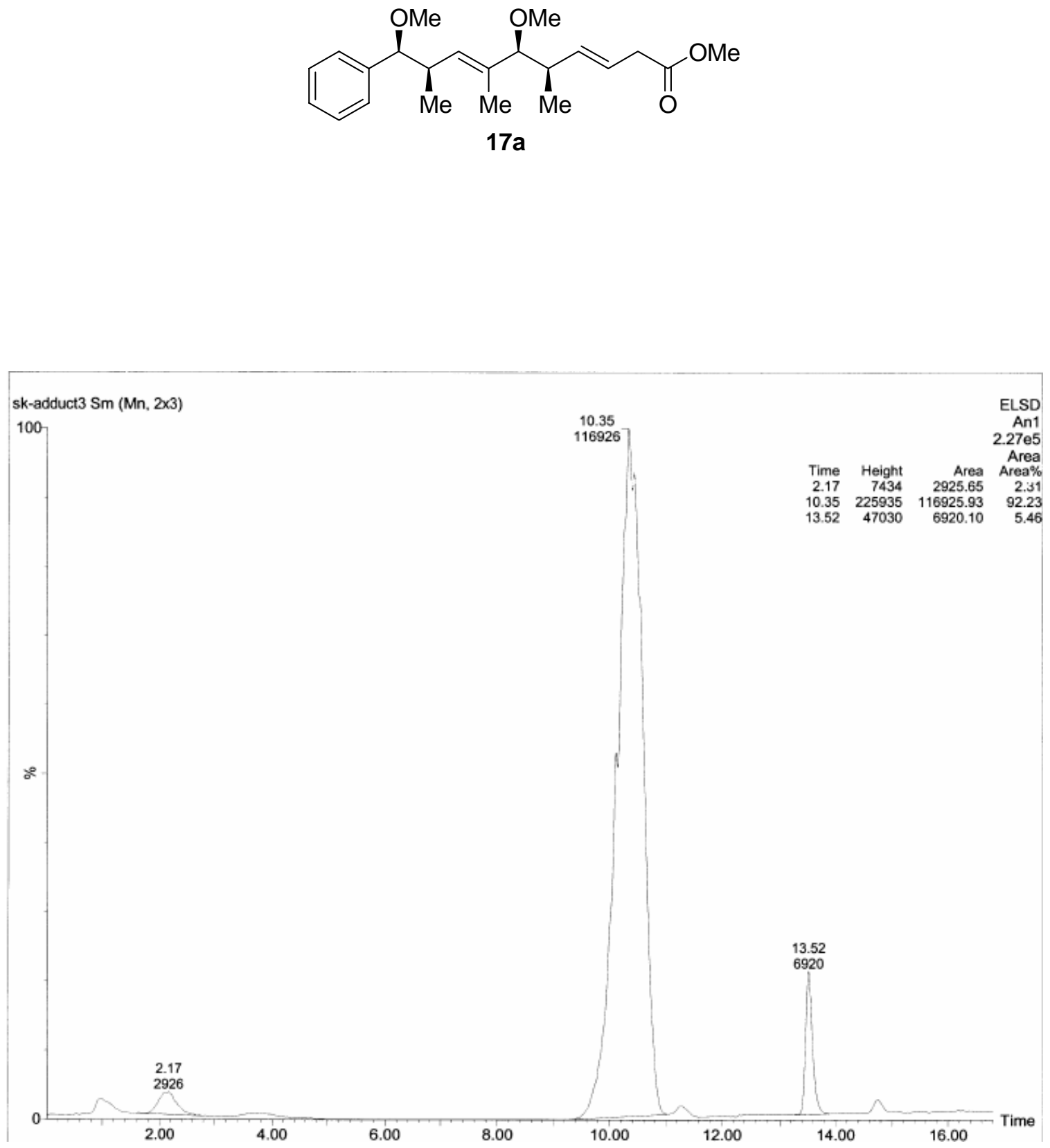


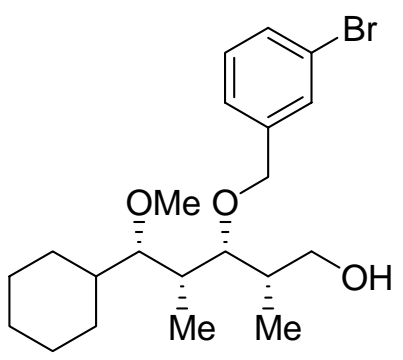

22

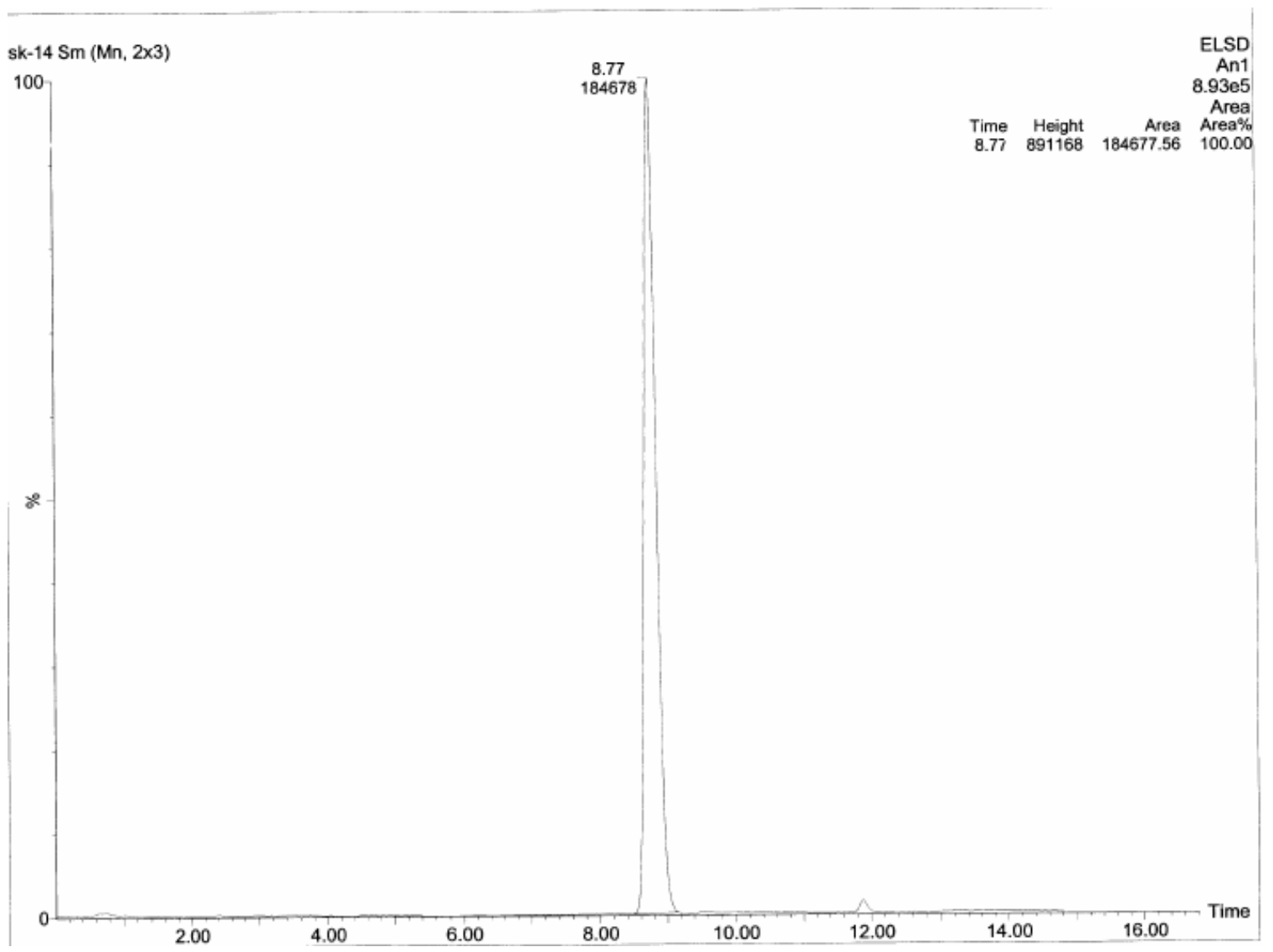




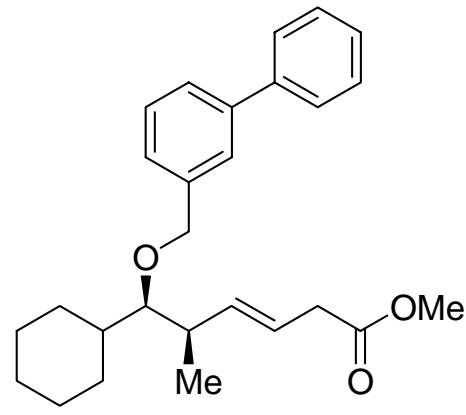

$25 a$

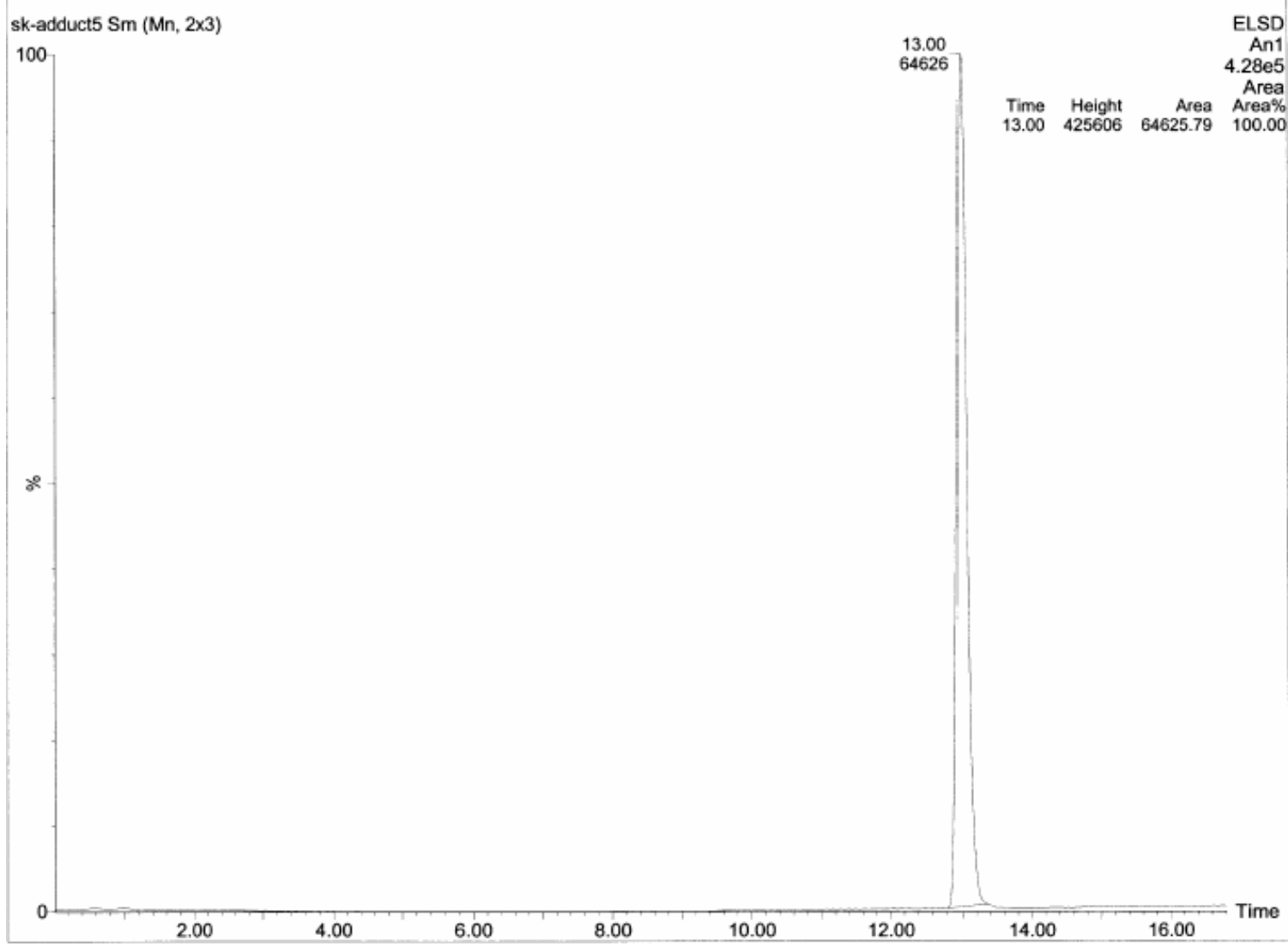




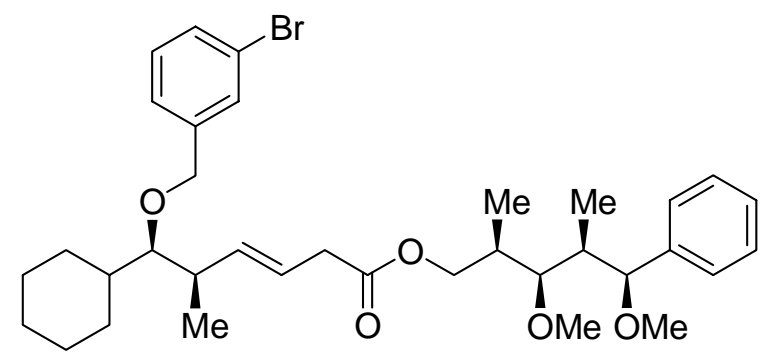

28

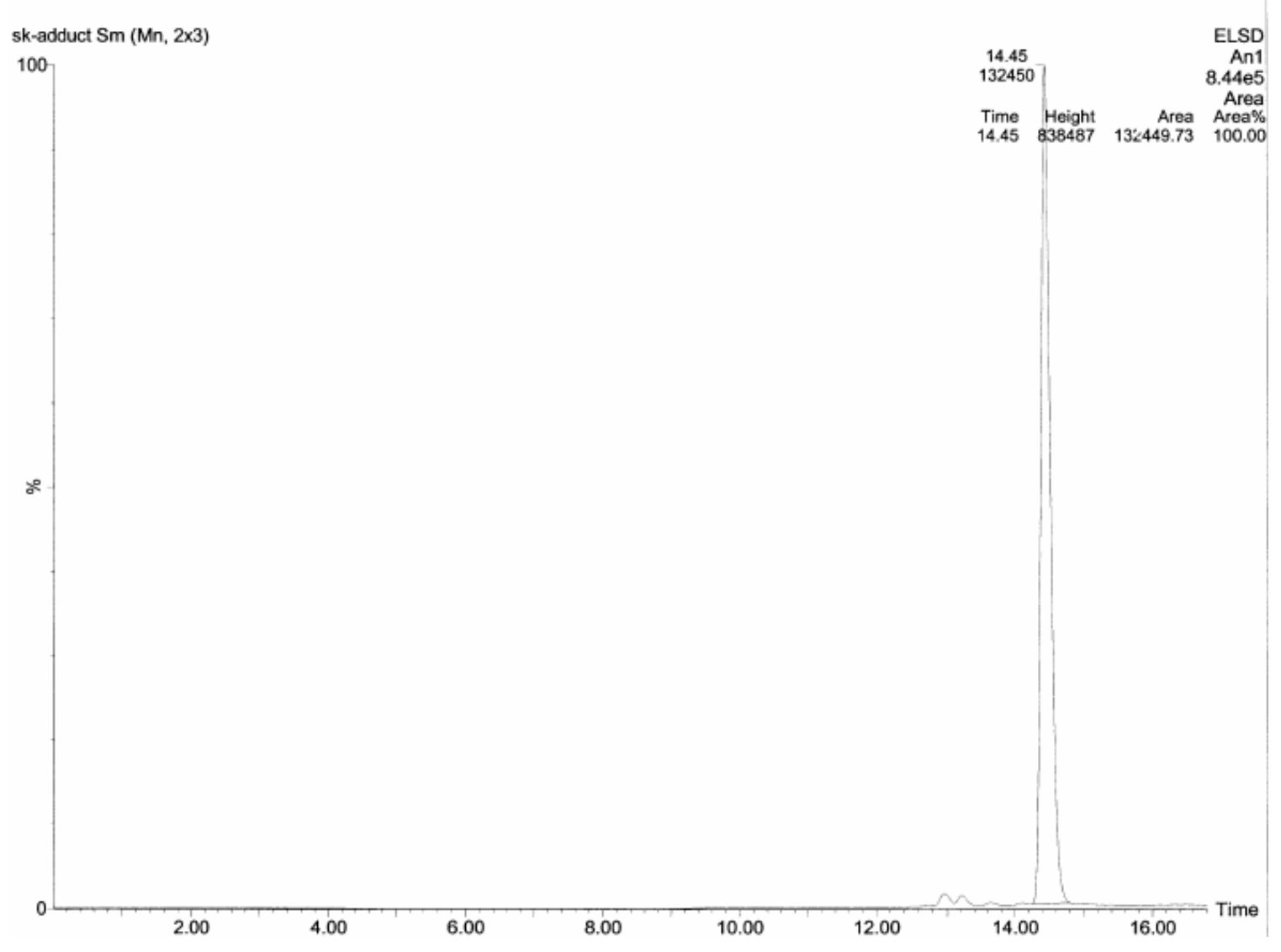

